\title{
Smart Multitask Bregman Clustering and Multitask Kernel Clustering
}

\author{
XIANCHAO ZHANG, XIAOTONG ZHANG, and HAN LIU, Dalian University of Technology
}

\begin{abstract}
Traditional clustering algorithms deal with a single clustering task on a single dataset. However, there are many related tasks in the real world, which motivates multitask clustering. Recently some multitask clustering algorithms have been proposed, and among them multitask Bregman clustering (MBC) is a very applicable method. MBC alternatively updates clusters and learns relationships between clusters of different tasks, and the two phases boost each other. However, the boosting does not always have positive effects on improving the clustering performance, it may also cause negative effects. Another issue of MBC is that it cannot deal with nonlinear separable data. In this article, we show that in MBC, the process of using cluster relationship to boost the cluster updating phase may cause negative effects, that is, cluster centroids may be skewed under some conditions. We propose a smart multitask Bregman clustering (S-MBC) algorithm which can identify the negative effects of the boosting and avoid the negative effects if they occur. We then propose a multitask kernel clustering (MKC) framework for nonlinear separable data by using a similar framework like MBC in the kernel space. We also propose a specific optimization method, which is quite different from that of MBC, to implement the MKC framework. Since MKC can also cause negative effects like MBC, we further extend the framework of MKC to a smart multitask kernel clustering (S-MKC) framework in a similar way that S-MBC is extended from MBC. We conduct experiments on 10 real world multitask clustering datasets to evaluate the performance of S-MBC and S-MKC. The results on clustering accuracy show that: (1) compared with the original MBC algorithm MBC, S-MBC and S-MKC perform much better; (2) compared with the convex discriminative multitask relationship clustering (DMTRC) algorithms DMTRC-L and DMTRC-R which also avoid negative transfer, S-MBC and S-MKC perform worse in the (ideal) case in which different tasks have the same cluster number and the empirical label marginal distribution in each task distributes evenly, but better or comparable in other (more general) cases. Moreover, S-MBC and S-MKC can work on the datasets in which different tasks have different number of clusters, violating the assumptions of DMTRC-L and DMTRC-R. The results on efficiency show that S-MBC and S-MKC consume more computational time than MBC and less computational time than DMTRC-L and DMTRC-R. Overall S-MBC and S-MKC are competitive compared with the state-of-the-art multitask clustering algorithms in synthetical terms of accuracy, efficiency and applicability.
\end{abstract}

Categories and Subject Descriptors: I.5.3 [Pattern Recognition]: Clustering

General Terms: Algorithms, Performance

Additional Key Words and Phrases: Multitask clustering, negative transfer, Bregman divergence, Mercer kernel

This work was supported by National Science Foundation of China (No. 61272374, 61300190). We wish to thank Xiaolei Zhang for providing the code of their DMTRC algorithm. We also thank the anonymous reviewers for their valuable comments.

A preliminary version of this article was presented in proceedings of the 27th AAAI conference (AAAI-13) [Zhang and Zhang 2013].

Authors' addresses: X. Zhang, X. Zhang, and H. Liu, School of Software, Dalian University of Technology, Dalian, 116024, China; emails: xczhang@dlut.edu.cn, zxt.dut@hotmail.com, liu.han.dut@gmail.com.

Permission to make digital or hard copies of all or part of this work for personal or classroom use is granted without fee provided that copies are not made or distributed for profit or commercial advantage and that copies bear this notice and the full citation on the first page. Copyrights for components of this work owned by others than ACM must be honored. Abstracting with credit is permitted. To copy otherwise, or republish, to post on servers or to redistribute to lists, requires prior specific permission and/or a fee. Request permissions from permissions@acm.org.

2015 Copyright is held by the owner/author(s). Publication rights licensed to ACM.

ACM 1556-4681/2015/07-ART8 $\$ 15.00$

DOI: http://dx.doi.org/10.1145/2747879 
ACM Reference Format:

Xianchao Zhang, Xiaotong Zhang and Han Liu. 2015. Smart multitask bregman clustering and multitask kernel clustering. ACM Trans. Knowl. Discov. Data 10, 1, Article 8 (July 2015), 29 pages.

DOI: http://dx.doi.org/10.1145/2747879

\section{INTRODUCTION}

Clustering is a fundamental problem in machine learning and data mining. Traditional clustering algorithms deal with a single clustering task on a single data set. However, there are many related tasks in real applications, such as web pages from different universities, slowly time-evolving datasets and so on, which motivates multitask clustering. Multi-task clustering is desired to improve the clustering performance of individual tasks through learning the relationship among related tasks.

Recently some multitask clustering algorithms have been proposed. Gu and Zhou [2009] proposed a cross domain multitask clustering framework by learning a subspace shared by all tasks. However, there is not a principled way to choose the dimensionality of the shared subspace. Moreover, it does not take the distribution of the shared subspace and geometric structure of the tasks into account. Gu et al. [2011] handled the problems earlier by learning a kernel among tasks, and their algorithm outperforms the one in Gu and Zhou [2009]. Nguyen et al. [2011] proposed a feature free and parameter light multitask clustering framework based on Kolmogorov complexity. Xie et al. [2012] proposed a multitask co-clustering algorithm by learning the relationships of features among different tasks. Zhang and Zhou [2012] proposed an algorithm which learns a shared subspace through domain adaptation. All of the methods earlier focus on crossdomain multitask clustering, which deals with an ideal situation that the related tasks have similar class labels. To deal with the case that there may be some outlier tasks or tasks with negative correlation, an unsupervised multitask learning method called convex DMTRC was proposed in Zhang [2015]. DMTRC can model both positive and negative task correlations by learning the intertask covariance matrix of the multivariate Gaussian prior. DMTRC has implementations in both the original space and nonlinear feature mapping space, which are called DMTRC-L and DMTRC-R, dealing with linear separable data and nonlinear separable data, respectively. However, the DMTRC method is based on the assumption that all tasks have the same number of clusters and the empirical label marginal distribution in each task distributes evenly, thus the applicability of DMTRC is limited. Unlike the methods mentioned earlier, Zhang and Zhang [2010] proposed a Bregman divergence based multitask clustering (MBC) algorithm, which can solve the multitask clustering problem when the related tasks are from a same distribution or similar distributions (the tasks share a set of data points). And it allows the cluster numbers of different tasks to be different. Thus $\mathrm{MBC}$ is very applicable in practice. MBC alternatively updates clusters and learns the relationships between clusters of different tasks, and the two phases boost each other. The alternative boosting was shown in Zhang and Zhang [2010] to gain better performance compared with single-task Bregman divergence clustering. However, the boosting of the second phase to the first phase does not always have positive effects on improving the clustering performance as desired, it may cause negative effects when the distributions of the tasks are not identical. Another issue of $\mathrm{MBC}$ is that it cannot deal with nonlinear separable data.

In this article, firstly, we show that using the cluster relationship to boost the cluster updating phase may cause negative effects, that is, for the data points not shared by other tasks, the centroids may be skewed. We then propose a S-MBC algorithm which identifies the negative effects of the boosting and avoids the negative effects if they occur. The basic idea of S-MBC is using a local loss of each task to measure whether the negative effects in this task occur, that is, the centroids deviate from the 
relatively ideal positions which have already been found. If the local loss of one task calculated by MBC is larger than the local loss calculated by the single-task Bregman divergence clustering, S-MBC will avoid using the boosting. Secondly, to deal with the multitask clustering of nonlinear separable data, we propose a MKC framework by using a similar framework like MBC in the kernel space, which is applicable to any kind of Mercer kernel. We also propose a specific optimization method, which is quite different from that of MBC, to implement the MKC framework. Since MKC is based on a similar framework like MBC, the boosting process of it can also cause negative effects. Therefore, we further extend the framework of MKC to a S-MKC framework in a similar way that S-MBC is extended from MBC, and implement the S-MKC framework. We conduct experiments on 10 real world multitask clustering datasets to evaluate the performance of S-MBC and S-MKC. The results on clustering accuracy show that: (1) compared with the original MBC algorithm MBC, S-MBC and S-MKC perform much better; (2) compared with the convex DMTRC algorithms DMTRC-L and DMTRC-R which also avoid negative transfer, S-MBC and S-MKC perform worse in the (ideal) case in which different tasks have the same cluster number and the empirical label marginal distribution in each task distributes evenly, but better or comparable in other (more general) cases. Moreover, S-MBC and S-MKC can work on datasets in which different tasks have different number of clusters, violating the assumptions of DMTRC$\mathrm{L}$ and DMTRC-R. The results on efficiency show that S-MBC and S-MKC consume more computational time than MBC and less computational time than DMTRC-L and DMTRC-R. Overall S-MBC and S-MKC are competitive compared with the state-ofthe-art multitask clustering algorithms in synthetical terms of accuracy, efficiency and applicability.

A preliminary version of this article was presented in proceedings of the 27th AAAI conference (AAAI-13). In this article, we extend the preliminary version from the following aspects: (1) we provide discussions of related concepts such that the article is self-contained; (2) we give a more detailed analysis about the negative effects of MBC; (3) we propose the MKC algorithm, and analyze that the negative effects also occur in MKC; (4) we provide detailed calculation processes of the proposed algorithms; (5) we prove the convergence of the proposed algorithms; (6) we provide an investigation of parameter settings empirically; (7) we conduct experiments on much more datasets, compare our proposed algorithms with DMTRC-L and DMTRC-R, and discuss the results in more details. (8) We investigate the computational time of MBC, DMTRC-L, DMTRC-R, our proposed algorithms S-MBC, MKC and S-MKC, and give some discussions.

The rest of this article is organized as follows. In Section 2, we provide some preliminary knowledge. In Section 3, we introduce the $\mathrm{MBC}$ algorithm and explain the negative effects. In Section 4, we propose the S-MBC algorithm. In Section 5, we propose the MKC algorithm. In Section 6, we propose the S-MKC algorithm. In Section 7, we show the experimental results. In Section 8, we review some related works. In Section 9, we draw the conclusion.

\section{PRELIMINARIES}

\subsection{Bregman Divergence}

Bregman divergence is introduced in Bregman [1967], it is a family of proximity functions sharing common properties. Recently, researchers have shown that many important algorithms can be generalized from Euclidean distance to metrics defined by Bregman divergence [Banerjee et al. 2005].

Definition 2.1 (Bregman Divergence). [Bregman 1967; Banerjee et al. 2005] Let $\phi: S \mapsto \mathbb{R}, S=\operatorname{dom}(\phi)$ be a strictly convex function defined on a convex set $S \subseteq \mathbb{R}^{d}$ 
Table I. Frequently Used Bregman Divergences

\begin{tabular}{|l|c|c|c|}
\hline Domain & $\phi(x)$ & $d_{\phi}(x, y)$ & Divergence \\
\hline $\mathbb{R}^{d}$ & $\|x\|^{2}$ & $\|x-y\|^{2}$ & Squared Euclidean distance \\
\hline $\mathbb{R}^{d}$ & $x^{T} A x$ & $(x-y)^{T} A(x-y)$ & Mahalanobis distance \\
\hline$d$-Simplex & $\sum_{j=1}^{d} x_{j} \log _{2} x_{j}$ & $\sum_{j=1}^{d} x_{j} \log _{2}\left(\frac{x_{j}}{y_{j}}\right)$ & KL divergence \\
\hline $\mathbb{R}_{++}$ & $-\log x$ & $\frac{x}{y}-\log \left(\frac{x}{y}\right)-1$ & Itakura-Saito distance \\
\hline
\end{tabular}

such that $\phi$ is differentiable on $\operatorname{ri}(S)$, which is assumed to be nonempty. The Bregman divergence $d_{\phi}: S \times \operatorname{ri}(S) \mapsto[0, \infty)$ is defined as

$$
d_{\phi}(x \| y)=\phi(x)-\phi(y)-\langle x-y, \nabla \phi(y)\rangle,
$$

where $\operatorname{dom}(\phi)$ denotes the effective domain of a function $\phi$, that is, the set of all $x$ such that $\phi(x)<+\infty, r i(S)$ denotes the relative interior of a set $S$, and $\nabla \phi(y)$ represents the gradient vector of $\phi$ evaluated at $y$.

The type of Bregman divergence is determined by the convex function $\phi$. We give some frequently used Bregman divergences and as well as their corresponding convex functions in Table I.

The literatures [Banerjee et al. 2005; Bregman 1967] give the details about the properties of Bregman divergence, and the clustering formulation with Bregman divergences can be seen in Banerjee et al. [2005].

\subsection{Mercer Kernel}

As the name implies, Mercer kernel is a kernel function that satisfy the Mercer condition. Nonlinear Mercer kernel such as Gaussian kernel performs a nonlinear data transformation into some high dimensional feature space that increases the probability of the linear separability of the data in the transformed space. It could be used to solve a variety of nonlinear optimization problems that arise in regression, classification, and clustering.

Theorem 2.2 [Characterization of Mercer kernel][Shawe-Taylor and Cristianini 2004]. Let $X$ be any input space and $K: X \times X \rightarrow \mathbb{R}$ be either continuous or have a finite domain. $K$ can be decomposed into a feature map $\phi$ into a Hilbert space $F$ applied to both its arguments followed by the evaluation of the inner product in $F$, that is, $K$ can be constructed as $K(x, y)=\langle\phi(x), \phi(y)\rangle_{F}$, if and only if it satisfies the finitely positive semi-definite property.

From Theorem 2.2, we can see that $K$ is a Mercer kernel if and only if the kernel matrix formed by restricting $K$ in any finite subset of input space $X$ to be positive semi-definite. Mercer kernels can be designed specifically for different data types, such as strings, graphs, and documents [Shawe-Taylor and Cristianini 2004]. In addition, new Mercer kernels can be built on existing ones. The detailed properties of Mercer kernels are given in Shawe-Taylor and Cristianini [2004]. We give some popular kernel functions in Table II.

\section{MULTITASK BREGMAN CLUSTERING AND ITS ISSUE}

\subsection{Problem Formulation}

Suppose we are given $T$ clustering tasks, each with a set of points, that is, $X^{(t)}=$ $\left\{x_{1}^{(t)}, x_{2}^{(t)}, \ldots, x_{n^{(t)}}^{(t)}\right\} \in R^{d \times n^{(t)}}, 1 \leq t \leq T$, where $n^{(t)}$ is the number of data points in the $t$ th task, and $X=\left\{X^{(1)}, \ldots, X^{(T)}\right\}$ denotes the datasets of all tasks. The dataset $X^{(t)}$ of each task is to be partitioned into $c^{(t)}$ clusters. For each task $t$, we need to find a partition 
Table II. Common Mercer Kernel Functions

\begin{tabular}{|c|c|l|}
\hline \multicolumn{1}{|c|}{ Kernel } & Kernel Function & \multicolumn{1}{c|}{ Parameters } \\
\hline Linear Kernel & $k(x, y)=x^{T} y+c$ & $c \in \mathbb{R}$ is the constant term \\
\hline Polynomial Kernel & $k(x, y)=\left(x^{T} y+c\right)^{d}$ & $\begin{array}{l}c \in \mathbb{R} \text { is the constant term, } \\
d \in \mathbb{N} \text { is the polynomial degree }\end{array}$ \\
\hline Gaussian Kernel & $K(x, y)=\exp \left(-\frac{\|x-y\|^{2}}{2 \sigma^{2}}\right)$ & $\begin{array}{l}\sigma \in \mathbb{R} \text { is the band width of the } \\
\text { Gaussian kernel }\end{array}$ \\
\hline Sigmoid Kernel & $K(x, y)=\tanh \left(\alpha x^{T} y+c\right)$ & $\begin{array}{l}\alpha \text { is the coefficient, } \\
c \in \mathbb{R} \text { is the constant term }\end{array}$ \\
\hline
\end{tabular}

$P^{(t)}=\left\{M^{(t)}, h^{(t)}\right\}$, which is defined by a set of centroids $M^{(t)}=\left\{m_{1}^{(t)}, \ldots, m_{c^{(t)}}^{(t)}\right\} \in R^{d \times c^{(t)}}$ and an assigning function $h^{(t)}: X^{(t)} \rightarrow\left\{1, \ldots, c^{(t)}\right\} . P=\left\{P^{(t)}\right\}_{t=1}^{T}$ denotes all the partitions, $M=\left\{M^{(t)}\right\}_{t=1}^{T}$ denotes the set of all the centroids, and $H=\left\{h^{(t)}\right\}_{t=1}^{T}$ denotes all the assigning functions. $d_{\phi}(x, y)$ denotes the Bregman divergence between data $x$ and $y$.

\subsection{Multitask Bregman Clustering}

Zhang and Zhang [2010] proposed a general framework for multitask clustering problem, which is called MBC, by learning a set of partitions $P$ to minimize the following objective function

$$
J=\frac{1}{T} \sum_{t=1}^{T} L^{(t)}\left(P^{(t)}, X^{(t)}\right)+\lambda \Omega(P) .
$$

In Equation (1), $L^{(t)}\left(P^{(t)}, X^{(t)}\right)=\frac{1}{n^{(t)}} \sum_{i=1}^{n^{(t)}} d_{\phi}\left(x_{i}^{(t)} \| m_{h^{(t)}\left(x_{i}^{(t)}\right)}^{(t)}\right)$ is a local loss for task $t$. $\Omega(P)=\frac{1}{T(T-1)} \sum_{t=1}^{T} \sum_{s=1, s \neq t}^{T} d\left(P^{(t)}, P^{(s)}\right)$ is a task regularization incorporating relationship among tasks. $\lambda \geq 0$ is a free parameter, which allows the loss function to be balanced between the local loss functions and the task regularization. $d\left(P^{(t)}, P^{(s)}\right)$ reflects the relationship between clusters of task $t$ and $s$, it is deduced from earth mover distance (EMD) [Rubner et al. 1998] and defined as

$$
\begin{array}{r}
d\left(P^{(t)}, P^{(s)}\right)=\min _{W^{t s}} \sum_{z=1}^{c^{(t)}} \sum_{l=1}^{c^{(s)}} w_{z l}^{t s} d_{\phi}\left(m_{z}^{(t)} \| m_{l}^{(s)}\right) \\
\text { s.t. } \quad \sum_{z=1}^{c^{(t)}} w_{z l}^{t s}=\pi_{l}^{s}, \sum_{l=1}^{c^{(s)}} w_{z l}^{t s}=\pi_{z}^{t}, w_{z l}^{t s} \geq 0, \forall z, l,
\end{array}
$$

where $W^{t s}$ is a nonnegative matrix of $\operatorname{size} c^{(t)} \times c^{(s)}$, and $w_{z l}^{t s}$ is the correlation coefficient between $m_{z}^{(t)}$ and $m_{l}^{(s)}$. In the constraints, $\pi_{z}^{t}=\frac{n_{z}^{(t)}}{n^{(t)}}$ is the proportion of cluster $z$ in the dataset $X^{(t)}$, and $\pi_{l}^{s}=\frac{n_{l}^{(s)}}{n^{(s)}}$ is the proportion of cluster $l$ in $X^{(s)}$. Obviously, $\sum_{z=1}^{c^{(t)}} \pi_{z}^{t}=$ $\sum_{s=1}^{c^{(s)}} \pi_{l}^{s}=1$, therefore $\sum_{z=1}^{c^{(t)}} \sum_{l=1}^{c^{(s)}} w_{z l}^{t s}=1$. $W^{t s}$ can be considered as a joint probability matrix between clusters of task $t$ and task $s$.

\subsection{Negative Effects of MBC}

It can be seen that there are two terms in Equation (1). The first term is to minimize the sum of Bregman divergence between the data points of all tasks and the corresponding centroid of each point. The second term is to minimize the difference between the partitions of any two tasks, it is designed to boost the clustering. The boosting may have positive effects, that is, helping the current cluster centroids approach the relatively 

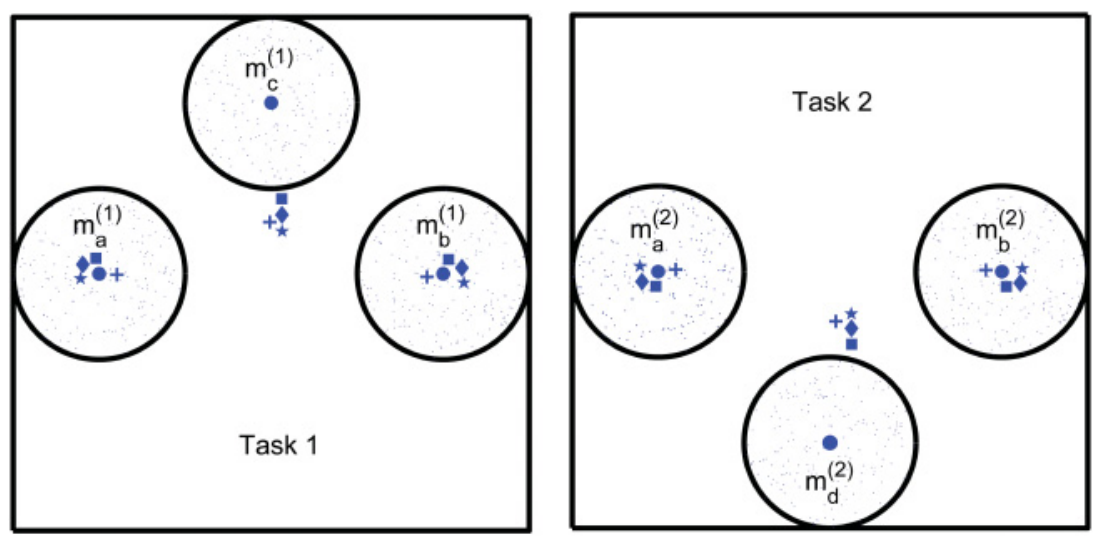

Fig. 1. An example of the negative effects. The initialized centroids of task 1 and task 2 are represented by "dot", and the recomputed centroids in the four iterations are represented by "plus", "square", "diamond", and "pentagram" in turn.

ideal positions. It may also have negative effects, that is, causing the current cluster centroids to deviate from the relatively ideal positions that have already been found. We analyze the negative effects as follows.

The difference is measured by a distribution metric EMD, which means that minimizing the difference between the partitions of any two tasks is equivalent to minimizing the difference between the distributions. Therefore, minimizing the second term is most applicable to the case that all the tasks are from a same distribution, but it cannot obtain a good clustering performance when the task distributions are different, which is a common case for multitask clustering.

Assume the Bregman divergence we use is squared Euclidean distance, Zhang and Zhang [2010] get the centroid $m_{z}^{(t)}$ by minimizing Equation (1)

$$
m_{z}^{(t)}=\frac{A \cdot u_{L}+B \cdot u_{R}}{A+B}
$$

where $A=\frac{n_{z}^{(t)}}{n^{(t)}}+\frac{\lambda}{c^{(t)}}, B=\frac{\lambda}{c^{(t)}}$, and

$$
\begin{aligned}
& u_{L}=\frac{1}{A}\left(\frac{1}{n^{(t)}} \sum_{i \in I_{z}^{(t)}} x_{i}^{(t)}+\frac{\lambda}{T-1} \sum_{s \neq t}^{T} \sum_{l=1}^{c^{(s)}} w_{z l}^{t s} m_{l}^{(s)}\right), \\
& u_{R}=(\nabla \phi)^{-1}\left(\frac{\lambda}{B \cdot(T-1)} \sum_{s \neq t}^{T} \sum_{l=1}^{c^{(s)}} w_{z l}^{t s} \nabla \phi\left(m_{l}^{(s)}\right)\right) .
\end{aligned}
$$

If we set $\lambda=0$, we can get $m_{z}^{(t)}=\frac{1}{n_{z}^{(t)}} \sum_{i \in I_{z}^{(t)}} x_{i}^{(t)}$, which is just the centroid calculated by $K$-means (KM). In Equation (3), MBC utilizes the relationship $w_{z l}^{t s}$ between the clusters of task $t$ and task $s$ to affect the centroid $m_{z}^{(t)}$ calculated by KM. Minimizing the second term has positive effects on the data points shared by other tasks to get their relatively ideal centroids, and negative effects on the data points that are not shared by other tasks.

We use two tasks whose data points are in a two-dimensional space (see task 1 and task 2 in Figure 1) as examples to illustrate the negative effects. Task 1 is composed of 
three clusters: $a, b$, and $c$, and task 2 is composed of three clusters: $a, b$, and $d$. Note that the two tasks share the data points in clusters $a$ and $b$. The data points in each cluster follow uniform distribution in a circle. We initialize the centroids of task 1 by the centers of three circles, denoted as $m_{a}^{(1)}, m_{b}^{(1)}$, and $m_{c}^{(1)}$, and initialize the centroids of task 2 by the centers of three circles, denoted as $m_{a}^{(2)}, m_{b}^{(2)}$, and $m_{d}^{(2)}$. It can be seen that the initial centroids are relatively ideal. We run the MBC algorithm 4 iterations which is convergent with convergence threshold 0.1 , the changes of the centroids of task 1 and task 2 in the four iterations are shown in Figure 1 with different shapes ("plus", "square", "diamond", and "pentagram"). As the second term of MBC need to minimize the difference among the partitions of all the tasks, we can get the probability matrix $W^{12}$ by Equationwith standard linear programming techniques to affect the centroids, the computed $W^{12}$ in the four iterations are as following.

$$
\begin{gathered}
W_{1}^{12}=\left(\begin{array}{lll}
0.2133 & 0.0000 & 0.1200 \\
0.0000 & 0.2133 & 0.1200 \\
0.1200 & 0.1200 & 0.0933
\end{array}\right) \\
W_{2}^{12}=W_{3}^{12}=W_{4}^{12}\left(\begin{array}{lll}
0.3333 & 0.0000 & 0.0000 \\
0.0000 & 0.3333 & 0.0000 \\
0.0000 & 0.0000 & 0.3333
\end{array}\right) .
\end{gathered}
$$

The centroids of task 1 and task 2 are recomputed according to $W^{12}$ and $W^{21}\left(W^{21}=\right.$ $\left(W^{12}\right)^{T}$ ) by Equation (3) in each iteration. In Figure 1, for the data points shared by task 1 and task 2 , that is, the data points in cluster $a$ and cluster $b, W^{12}$ and $W^{21}$ help them to find the relatively ideal centroids (the recomputed centroids $m_{a}^{(1)}, m_{b}^{(1)}$, $m_{a}^{(2)}$, and $m_{b}^{(2)}$ in the four iterations are all around the relatively ideal centroids, that is, the centers of their respective circles). While for the data points in cluster $c$ and cluster $d, W^{12}$ and $W^{21}$ make their centroids deviate far away from the relatively ideal positions which have already been found (they converge at the positions outside the circles). Obviously, the $W^{12}$ in Equation (4) is not a real good result for helping find the relatively ideal centroids. For example, the weights between the centroids of cluster $c$ in task 1 and cluster $a, b, d$ in task 2 in $W_{1}^{12}$ should be 0 , since there are no relationships between cluster $c$ in task 1 and clusters $a, b, d$ in task 2 . However, the weights in $W_{1}^{12}$ are $0.1200,0.1200,0.0933$, respectively. Thus, they will influence each other because of the constraints in Equation (2), which will bring negative effects to the relatively ideal centroids selection.

\section{SMART MULTITASK BREGMAN CLUSTERING}

\subsection{Avoiding the Negative Effects of MBC}

The most urgent work is to judge whether the negative effects occur. Then when it happens, we can adjust the centroids at once. The famous clustering algorithm KM uses the condition that the sum of the squared error is convergent to decide whether the clustering is finished. For the same reason, we compare the local loss of each task calculated by MBC with the local loss calculated by SBC (single-task Bregman divergence clustering algorithm) to judge whether the negative effects occur.

Specifically, in each iteration, we firstly compute the corresponding centroids set $M^{(t)}$ and assigning function $h^{(t)}$ through SBC (without task regularization) and MBC (considering task regularization) respectively for each task $t$. Secondly, we calculate the local loss function in Equation (1) with $M^{(t)}$ and $h^{(t)}$ of both methods. Thirdly, we choose the $M^{(t)}$ and $h^{(t)}$ whose corresponding method has a smaller local loss. Then, we 
recompute the $M^{(t)}$ and $h^{(t)}$ iteratively until $J_{s t}=\frac{1}{T} \sum_{t=1}^{T} L^{(t)}\left(P^{(t)}, X^{(t)}\right)$ converges to a stable state. In this way, we can prevent centroids skewing caused by MBC and get the relatively ideal centroids. We call the algorithm with the negative effects avoiding scheme S-MBC.

\subsection{Optimization}

In this section, we consider the optimization problem of the S-MBC algorithm. The literature [Zhang and Zhang 2010] provides a detailed explanation about the optimization of the MBC algorithm, which is part of the S-MBC optimization process. As we need to compute the $M^{(t)}$ calculated by MBC, we first rewrite the framework of MBC in its elemental form in Equation (5).

$$
\begin{aligned}
\min _{P, W} J= & \sum_{t=1}^{T} \frac{1}{n^{(t)}} \sum_{i=1}^{n^{(t)}} d_{\phi}\left(x_{i}^{(t)} \| m_{h^{(t)}\left(x_{i}^{(t)}\right)}^{(t)}\right) \\
& +\frac{\lambda}{T-1} \sum_{t \neq s}^{T} \sum_{z=1}^{c^{(t)}} \sum_{l=1}^{c^{(s)}}\left(w_{z l}^{t s} d_{\phi}\left(m_{z}^{(t)} \| m_{l}^{(s)}\right)+w_{l z}^{s t} d_{\phi}\left(m_{l}^{(s)} \| m_{z}^{(t)}\right)\right) \\
& \text { s.t. } \sum_{z=1}^{c^{(t)}} w_{z l}^{t s}=\pi_{l}^{s}, \sum_{l=1}^{c^{(s)}} w_{z l}^{t s}=\pi_{z}^{t}, w_{z l}^{t s} \geq 0, \forall z, l .
\end{aligned}
$$

In Equation (5), $\pi_{z}^{t}=\frac{n_{z}^{(t)}}{n^{(t)}}, \pi_{l}^{s}=\frac{n_{l}^{(s)}}{n^{(s)}}$ are determined by $h^{(t)}$ and $h^{(s)}$ respectively, the two series of equality constraints $W^{t s}$ and $\left\{h^{(t)}, h^{(s)}\right\}$ make the optimization problem difficult. Since the two equality constraints aim to prevent trivial solutions with very unbalanced clusters, we simply relax $\pi_{z}^{t}=\frac{1}{c^{(t)}}$ and $\pi_{l}^{s}=\frac{1}{c^{(s)}}$. In the optimization process, we relax the hard assignment function into continuous nonnegative continuous value, which can lead to an approximate solution [Mørup and Hansen [2009]. Then the assigning function for each task $h^{(t)}$ turns to be $H^{(t)} \in R^{c^{(t)} \times n^{(t)}}$ with constraint $H^{(t)} \geq 0$.

It can be seen that minimizing Equation (5) is with respect to the relation matrices $W=\left\{W^{t s}\right\}$, the cluster centroids $M$ and assigning functions $H=\left\{H^{(t)}\right\}_{t=1}^{T}$, the computation of the three variable groups is described in the following section.

Computation of $\boldsymbol{W}$ : Given $M$ and $H$, each matrix $W^{t s}$ is independently determined by Equation (2). This problem can be easily solved by standard linear programming techniques. It will lead to such a solution that $w_{z l}^{t s}$ is large if $d_{\phi}\left(m_{z}^{(t)} \| m_{l}^{(s)}\right)$ is small. $w_{z l}^{t s}$ here measures the similarity between cluster $z$ of task $t$ and cluster $l$ of task $s$.

\section{Computation of $M$ :}

(1) We first consider the situation when the clustering centroids $M=\left\{M^{(t)}\right\}_{t=1}^{T}$ are obtained with the task regularization. Given $W$ and $H$, clustering centroids $M^{(t)}$ is determined by

$$
\begin{aligned}
\min _{M^{(t)}}= & \frac{1}{n^{(t)}} \sum_{i=1}^{n^{(t)}} \sum_{z=1}^{c^{(t)}} H_{z i}^{(t)} d_{\phi}\left(x_{i}^{(t)} \| m_{z}^{(t)}\right) \\
& +\frac{\lambda}{T-1} \sum_{s \neq t}^{T} \sum_{z=1}^{c^{(t)}} \sum_{l=1}^{c^{(s)}}\left(w_{z l}^{t s} d_{\phi}\left(m_{z}^{(t)} \| m_{l}^{(s)}\right)+w_{l z}^{s t} d_{\phi}\left(m_{l}^{(s)} \| m_{z}^{(t)}\right)\right) .
\end{aligned}
$$


We can find that each centroid $m_{z}^{(t)}$ in $M^{(t)}$ is independently determined by

$$
\begin{aligned}
\min _{m_{z}^{(t)}}= & \frac{1}{n^{(t)}} \sum_{i=1}^{n^{(t)}} H_{z i}^{(t)} d_{\phi}\left(x_{i}^{(t)} \| m_{z}^{(t)}\right) \\
& +\frac{\lambda}{T-1} \sum_{s \neq t}^{T} \sum_{l=1}^{c^{(s)}}\left(w_{z l}^{t s} d_{\phi}\left(m_{z}^{(t)} \| m_{l}^{(s)}\right)+w_{l z}^{s t} d_{\phi}\left(m_{l}^{(s)} \| m_{z}^{(t)}\right)\right) .
\end{aligned}
$$

With the following two properties of Bregman divergences mentioned in Zhang and Zhang [2010], the problem can be easily solved. Let $\omega_{i} \geq 0$, and $\sum_{i} \omega_{i}>0$, then

Theorem 4.1 [Zhang And Zhang 2010]. $\sum_{i=1}^{n} w_{i} d_{\phi}\left(x_{i} \| \theta\right)=A d_{\phi}\left(u_{L} \| \theta\right)+C$, where $A=$ $\sum_{i=1}^{n} w_{i}, u_{L}=\sum_{i=1}^{n} w_{i} x_{i} / A, C$ is a constant w.r.t $\theta$.

Theorem 4.2 [Zhang And Zhang 2010]. $\sum_{i=1}^{n} w_{i} d_{\phi}\left(\theta \| x_{i}\right)=A d_{\phi}\left(\theta \| u_{R}\right)+C$, where $A=\sum_{i=1}^{n} w_{i}, u_{R}=(\nabla \phi)^{-1}\left(\sum_{i=1}^{n} w_{i} \nabla \phi\left(x_{i}\right) / A\right)$, and $C$ is a constant w.r.t $\theta$.

Applying the two theorems earlier, Equation (7) turns to be

$$
\min _{m_{z}^{(t)}} A \cdot d_{\phi}\left(u_{L} \| m_{z}^{(t)}\right)+B \cdot d_{\phi}\left(m_{z}^{(t)} \| u_{R}\right)
$$

where $A, B, u_{L}$, and $u_{R}$ are the same as those in Equation (3).

Some Bregman divergences are symmetric, such as squared Euclidean distance, and others are asymmetric, such as KL divergence, so there are two cases to compute the centroid $m_{z}^{(t)}$.

—when $d_{\phi}(x \| y)$ is symmetric, applying Theorem 4.1 again, Equation (8) turns out to be

$$
(A+B) d_{\phi}\left(\frac{A \cdot u_{L}+B \cdot u_{R}}{A+B} \| m_{z}^{(t)}\right)
$$

and we obtain $m_{z}^{(t)}=\frac{A \cdot u_{L}+B \cdot u_{R}}{A+B}$.

- when $d_{\phi}(x \| y)$ is asymmetric, a closed form of minimum solution could not be obtained. However, the article [Nielsen and Nock 2009] provided an efficient Geodesic-walk dichotomic approximation algorithm for this problem.

(2) When the clustering centroids $M$ are obtained without the task regularization, optimizing $m_{z}^{(t)}$ is equivalent to minimize Equation (7) with $\lambda=0$. The centroid $m_{z}^{(t)}$ is

$$
m_{z}^{(t)}=\frac{1}{\sum_{i=1}^{n^{(t)}} H_{z i}^{(t)}} \sum_{i=1}^{n^{(t)}} H_{z i}^{(t)} x_{i}^{(t)} .
$$

Computation of $\boldsymbol{H}$ : Given $M$ and $W$, each assigning function $H^{(t)}$ is independently determined by $\min _{H^{(t)}}=\sum_{i=1}^{n^{(t)}} \sum_{z=1}^{c^{(t)}} H_{z i} d_{\phi}\left(x_{i}^{(t)} \| m_{z}^{(t)}\right)$. Then, according to Dhillon and Sra [2005], we obtain

$$
H_{z i}^{(t)} \leftarrow H_{z i}^{(t)} \frac{\left[\left(M^{(t)}\right)^{T}\left(\zeta\left(M^{(t)} H^{(t)}\right) \odot X^{(t)}\right)\right]_{z i}}{\left[\left(M^{(t)}\right)^{T}\left(\zeta\left(M^{(t)} H^{(t)}\right) \odot M^{(t)} H^{(t)}\right)\right]_{z i}},
$$

where $\zeta(x)$ denotes $\nabla^{2}(x)$.

The overall process is listed in Algorithm 1. 


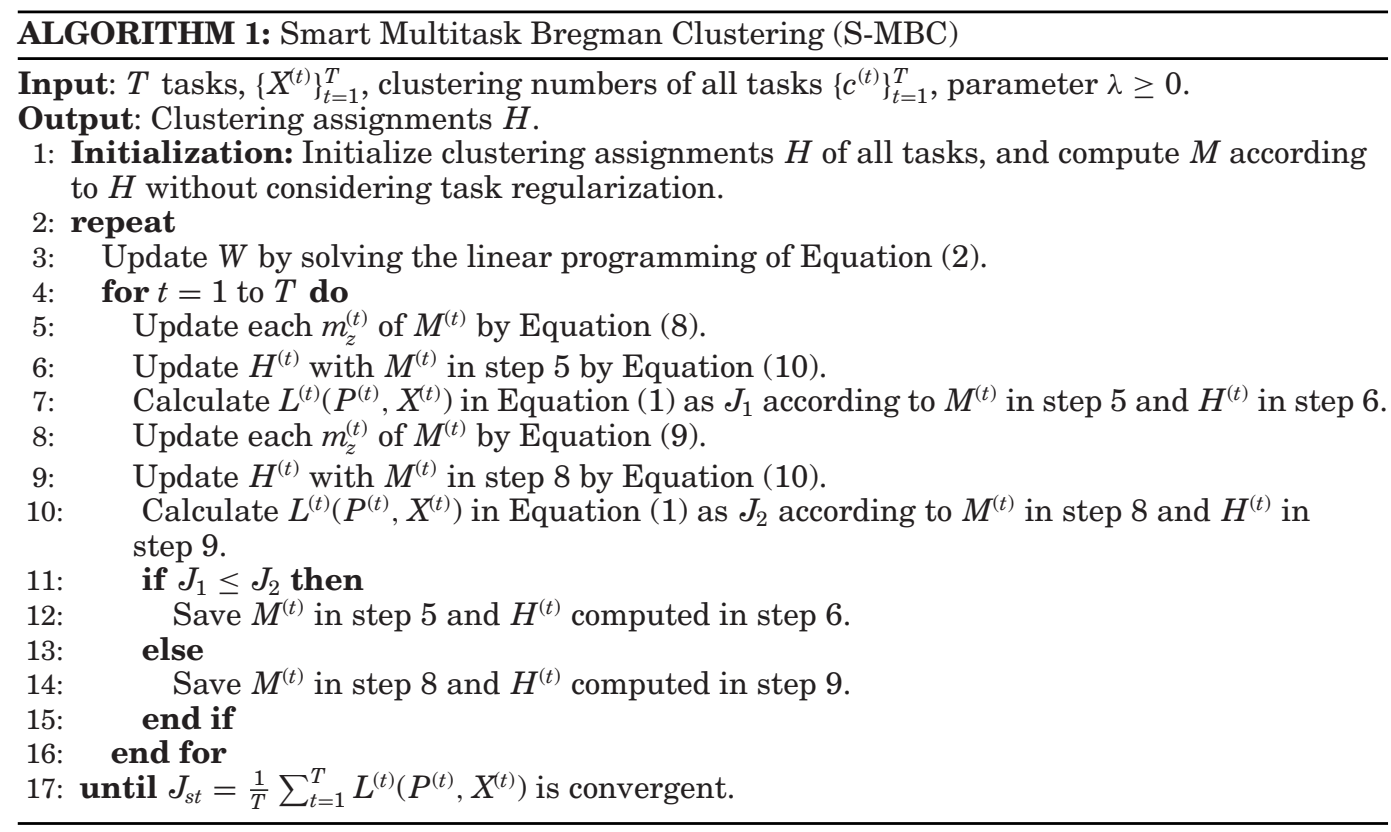

\subsection{Convergence Analysis of S-MBC}

LEMMA 4.3 [BANERJEE ET AL. 2005]. Let $X$ be a random variable that take values in $X=\left\{x_{i}\right\}_{i=1}^{n} \subset S \subseteq R^{d}$ following a positive probability measure $v$ such that $E_{v}[X] \in \operatorname{ri}(S)$. Given a Bregman divergence $d_{\phi}: S \times r i(S) \mapsto[0, \infty)$, the problem $\min _{s \in r i(S)} E_{v}\left[d_{\phi}(X|| s)\right]=$ $\sum_{i=1}^{n} v_{i} d_{\phi}\left(x_{i} \| s\right)$ has a unique solution given by $s^{\dagger}=\mu=E_{v}[X]$.

TheOREM 4.4. Algorithm 1 monotonically decreases the objective function $J_{s t}$.

Proof. It can be seen that minimizing the objective function $J_{s t}=$ $\frac{1}{T} \sum_{t=1}^{T} L^{(t)}\left(P^{(t)}, X^{(t)}\right)$ is with respect to $H^{(t)}$ and $M^{(t)}$. For $H^{(t)}$, the convergence property of $J_{s t}$ has been proved in Dhillon and Sra [2005].

For $M^{(t)}$, there are two updating rules in Equations (8) and (9). Denote the objective value under iteration $i$ as $J_{s t}^{i}$, the objective value in the next iteration $i+1$ as

$$
J_{s t}^{i+1}=\left\{\begin{array}{l}
J_{s t}\left(M_{e q .(8)}^{(t)}, H^{(t)}\right) \text { if } M^{(t)} \text { is computed by Eq. (8). } \\
J_{s t}\left(M_{e q .(9)}^{(t)}, H^{(t)}\right) \text { if } M^{(t)} \text { is computed by Eq. (9). }
\end{array}\right.
$$

We first analyze the $M^{(t)}$ updated by Equation (9). For each $m_{z}^{(t)}$ in $M^{(t)}$, minimizing $J_{s t}$ turns to be $J\left(m_{z}^{(t)}\right)=\frac{1}{n^{(t)}} \sum_{i=1}^{n^{(t)}} H_{z i} d_{\phi}\left(x_{i}^{(t)} \| m_{z}^{(t)}\right)$. We can get the centroid $m_{z}^{(t)}$ computed by Equation (9) based on Lemma 4.3, thus $J_{s t}$ can monotonically decrease with $M^{(t)}$ updated by Equation (9), that is, $J_{s t}^{i+1}=J_{s t}\left(M_{e q .(9)}^{(t)}, H^{(t)}\right)<J_{s t}^{i}$.

We further prove that updating $M^{(t)}$ by Equation (8) can also monotonically decrease the value of $J_{s t}$. Since the objective value is the smaller one of $J_{s t}^{i+1}$, that is, if $J_{s t}\left(M_{e q .(8)}^{(t)}, H^{(t)}\right) \leq J_{s t}\left(M_{e q .(9)}^{(t)}, H^{(t)}\right)$, then $J_{s t}^{i+1}=J_{s t}\left(M_{e q .(8)}^{(t)}, H^{(t)}\right) \leq J_{s t}\left(M_{e q .(9)}^{(t)}, H^{(t)}\right)<J_{s t}^{i}$, otherwise $J_{s t}^{i+1}=J_{s t}\left(M_{e q .(9)}^{(t)}, H^{(t)}\right)<J_{s t}^{i}$. 
Thus, the computation of $M^{(t)}$ by either Equations (8) or (9) can monotonically decrease the value of $J_{s t}$.

From the previous analysis, and since $J_{s t} \geq 0$, Algorithm 1 is guaranteed to converge.

\section{MULTITASK KERNEL CLUSTERING}

In this section, we introduce a MKC algorithm for nonlinear separable data, which can apply to any kind of Mercer kernel [Saunders et al. 1998].

\subsection{Problem Formulation}

Suppose we are given $T$ clustering tasks, each with a set of points, that is, $X^{(t)}=$ $\left\{x_{1}^{(t)}, x_{2}^{(t)}, \ldots, x_{n^{(t)}}^{(t)}\right\} \in R^{d \times n^{(t)}}, 1 \leq t \leq T$, where $n^{(t)}$ is the number of data points in the $t$ th task, and $X=\left\{X^{(1)}, \ldots, X^{(T)}\right\}$ denotes the datasets of all tasks. The dataset $X^{(t)}$ of each task is to be partitioned into $c^{(t)}$ clusters. For each task $t$, we need to find a partition $P^{(t)}=\left\{M^{(t)}, Z^{(t)}\right\}$, which is defined by a set of centroids $M^{(t)}=\left\{m_{1}^{(t)}, \ldots, m_{c^{(t)}}^{(t)}\right\} \in R^{d \times c^{(t)}}$ and a partition matrix $Z^{(t)} \in\{0,1\}^{n^{(t)} \times c^{(t)}} . P=\left\{P^{(t)}\right\}_{t=1}^{T}$ denotes all the partitions, $M=$ $\left\{M^{(t)}\right\}_{t=1}^{T}$ denotes the set of all the centroids, and $Z=\left\{Z^{(t)}\right\}_{t=1}^{T}$ denotes all the partition matrices. We consider a nonlinear mapping $\phi$ from the original feature space to the feature space $F$, that is, $\phi: R^{d} \rightarrow F$, the inner product in $F$ is defined as $\langle\phi(x), \phi(y)\rangle_{F}=$ $K(x, y)$. Then for each task $t, X^{(t)}$ mapped in $F$ is defined as $\phi\left(\mathrm{X}^{(t)}\right)=\left\{\phi\left(x_{1}^{(t)}\right), \ldots, \phi\left(x_{n^{(t)}}^{(t)}\right)\right\}$, $1 \leq t \leq T$.

\subsection{Objective Function}

Let us consider the case of single-task Kernel $K$-means (KKM) clustering first. We take the $t$ th task for example. We are going to partition the $t$ th dataset into $c^{(t)}$ clusters. The classical KKM algorithm achieves this goal by minimizing the following objective function.

$$
J_{t}=\frac{1}{n^{(t)}} \sum_{k=1}^{c^{(t)}} \sum_{i=1}^{n^{(t)}} Z_{i k}^{(t)}\left\|\phi\left(x_{i}^{(t)}\right)-m_{k}^{(t)}\right\|_{2}^{2},
$$

where $\|\cdot\|_{2}$ is 2 -norm. Equation (11) can be rewritten as

$$
\begin{gathered}
J_{t}=\frac{1}{n^{(t)}}\left\|\phi\left(X^{(t)}\right)-M^{(t)} Z^{(t) T}\right\|_{F}^{2} \\
\text { s.t. } \quad Z^{(t)} \in\{0,1\}^{n^{(t)} \times c^{(t)}},
\end{gathered}
$$

where $\|\cdot\|_{F}$ is a Frobenius norm, $Z^{(t)} \in\{0,1\}^{n^{(t)} \times c^{(t)}}$ is a partition matrix, which represents the clustering assignment, such that $Z_{i k}^{(t)}=1$ if $x_{i}^{(t)}$ belongs to cluster $k$ of task $t$, and $Z_{i k}^{(t)}=0$ otherwise.

In the kernel space, we use a multitask framework similar to that in Equation (1), the difference is that the local loss function is now defined as

$$
L^{(t)}\left(P^{(t)}, X^{(t)}\right)=\frac{1}{n^{(t)}}\left\|\phi\left(X^{(t)}\right)-M^{(t)} Z^{(t) T}\right\|_{F}^{2} .
$$

And the task regularization is defined as

$$
\Omega(P)=\frac{1}{T(T-1)} \sum_{t=1}^{T} \sum_{s=1, s \neq t}^{T} d\left(P^{(t)}, P^{(s)}\right),
$$


where $d\left(P^{(t)}, P^{(s)}\right)$ can be defined as

$$
\begin{array}{r}
d\left(P^{(t)}, P^{(s)}\right)=\min _{W^{t s}} \sum_{z=1}^{c^{(t)}} \sum_{l=1}^{c^{(s)}} w_{z l}^{t s}\left\|m_{z}^{(t)}-m_{l}^{(s)}\right\|_{2}^{2} \\
\text { s.t. } \quad \sum_{z=1}^{c^{(t)}} w_{z l}^{t s}=\pi_{l}^{s}, \sum_{l=1}^{c^{(s)}} w_{z l}^{t s}=\pi_{z}^{t}, w_{z l}^{t s} \geq 0, \forall z, l .
\end{array}
$$

\subsection{Optimization}

By its definition, the elements in $Z^{(t)}$ can only take binary values, which makes the minimization in Equation (1) very difficult. However, the literature [Ding et al. 2010] proves that $Z^{(t)}$ can be relaxed into nonnegative continuous domain. Thus, according to Equations (13-15), by relaxing $\pi_{z}^{t}=\frac{1}{c^{(t)}}$ and $\pi_{l}^{s}=\frac{1}{c^{(s)}}$ like the MBC algorithm, we can get the following optimization problem of MKC.

$$
\begin{gathered}
\min _{P, W} J=\sum_{t=1}^{T} \frac{1}{n^{(t)}}\left\|\phi\left(X^{(t)}\right)-M^{(t)} Z^{(t) T}\right\|_{F}^{2}+\frac{2 \lambda}{(T-1)} \sum_{t \neq s}^{T} \sum_{z=1}^{c^{(t)}} \sum_{l=1}^{c^{(s)}} w_{z l}^{t s}\left\|m_{z}^{(t)}-m_{l}^{(s)}\right\|_{2}^{2} \\
\text { s.t. } \quad \lambda \geq 0, Z^{(t)} \geq 0, \sum_{z=1}^{c^{(t)}} w_{z l}^{t s}=\frac{1}{c^{(s)}}, \sum_{l=1}^{c^{(s)}} w_{z l}^{t s}=\frac{1}{c^{(t)}}, w_{z l}^{t s} \geq 0 .
\end{gathered}
$$

It can be seen that minimizing Equation (16) is with respect to three groups of variables, that is, cluster centroids $M$, partition matrices $Z$, and relation matrices $W=\left\{W^{t s}\right\}$.

Computation of $\boldsymbol{M}$ : Given $Z$ and $W$, since the cluster centroids are obtained with the task regularization, optimizing Equation (16) with respect to $M$ is equivalent to optimizing

$$
\begin{aligned}
& \min \sum_{t=1}^{T} \frac{1}{n^{(t)}}\left\|\phi\left(X^{(t)}\right)-M^{(t)} Z^{(t) T}\right\|_{F}^{2}+\frac{2 \lambda}{(T-1)} \sum_{t \neq s}^{T}\left(\operatorname{tr}\left(E^{(t)}\left(M^{(t)}\right)^{T}\left(M^{(t)}\right)\right)\right. \\
& \left.\quad+\operatorname{tr}\left(E^{(s)}\left(M^{(s)}\right)^{T}\left(M^{(s)}\right)\right)-2 \operatorname{tr}\left(W^{t s}\left(M^{(s)}\right)^{T}\left(M^{(t)}\right)\right)\right),
\end{aligned}
$$

where $E^{(t)}$ is a diagonal matrix with $E_{i i}^{(t)}=\frac{1}{c^{(t)}}\left(i=1, \ldots, c^{(t)}\right)$.

Fixing $\left\{M^{(s)}\right\}_{s \neq t}^{T}$, optimizing Equation (17) with respect to $M^{(t)}$ is equivalent to optimizing

$$
\begin{aligned}
J_{1}= & \frac{1}{n^{(t)}}\left\|\phi\left(X^{(t)}\right)-M^{(t)} Z^{(t) T}\right\|_{F}^{2}+\frac{2 \lambda}{T-1}\left((T-1) \operatorname{tr}\left(E^{(t)}\left(M^{(t)}\right)^{T} M^{(t)}\right)\right. \\
& +\sum_{\mathrm{s} \neq t}^{T}\left(\operatorname{tr}\left(E^{(s)}\left(M^{(s)}\right)^{T} M^{(s)}\right)-2 \operatorname{tr}\left(W^{t s}\left(M^{(t)}\right)^{T} M^{(s)}\right)\right)
\end{aligned}
$$

Setting $\frac{\partial J_{1}}{\partial M^{(t)}}=0$, we obtain

$$
M^{(t)}=\left(\frac{1}{n^{(t)}} \phi\left(X^{(t)}\right) Z^{(t)}+\frac{2 \lambda}{T-1} \sum_{s \neq t}^{T} M^{(s)} W^{s t}\right)\left(\frac{1}{n^{(t)}} Z^{(t) T} Z^{(t)}+2 \lambda E^{(t)}\right)^{-1}
$$


In Equation (19), $\phi\left(X^{(t)}\right)$ and $M^{(s)}$ are unknown, however, $\phi\left(X^{(s)}\right)^{T} M^{(t)}$ and $\left(M^{(s)}\right)^{T} M^{(t)}$ can be calculated. Setting $F^{(s, t)}=\phi\left(X^{(s)}\right)^{T} M^{(t)}, G^{(s, t)}=\left(M^{(s)}\right)^{T} M^{(t)}(t, s=1, \ldots, T)$, then optimizing $M^{(t)}$ is equivalent to optimizing $F^{(s, t)}$ and $G^{(s, t)}(s=1, \ldots, T)$.

Setting $K^{(s, t)}=\phi\left(X^{(s)}\right)^{T} \phi\left(X^{(t)}\right)$, we obtain

$$
\begin{gathered}
F^{(s, t) \text { new }}=\left(\frac{1}{n^{(t)}} K^{(s, t)} Z^{(t)}+\frac{2 \lambda}{T-1} \sum_{p \neq t}^{T} F^{(s, p)} W^{p t}\right)\left(\frac{1}{n^{(t)}} Z^{(t) T} Z^{(t)}+2 \lambda E^{(t)}\right)^{-1} . \\
G^{(s, t) \text { new }}=\left(\frac{1}{n^{(s)}} Z^{(s) T} Z^{(s)}+2 \lambda E^{(s)}\right)^{-1} V\left(\frac{1}{n^{(t)}} Z^{(t) T} Z^{(t)}+2 \lambda E^{(t)}\right)^{-1},
\end{gathered}
$$

where $V=\frac{1}{n^{(s)} n^{(t)}} Z^{(s) T} K^{(s, t)} Z^{(t)}+\frac{2 \lambda}{n^{(s)}(T-1)} \sum_{p \neq t}^{T} Z^{(s) T} F^{(s, p)} W^{p t}$

$$
+\frac{2 \lambda}{n^{(t)}(T-1)} \sum_{q \neq s}^{T} W^{s q} F^{(t, q) T} Z^{(t)}+\frac{4 \lambda^{2}}{(T-1)^{2}} \sum_{q \neq s}^{T} \sum_{p \neq t}^{T} W^{s q} G^{(q, p)} W^{p t} .
$$

Computation of $\boldsymbol{Z}$ : Given $M$ and $W$, each $Z^{(t)}$ can be obtained by optimizing

$$
\begin{aligned}
& \min \left\|\phi\left(X^{(t)}\right)-M^{(t)} Z^{(t) T}\right\|_{F}^{2} \\
& \text { s.t. } \quad Z^{(t)} \geq 0 .
\end{aligned}
$$

For the constraint $Z^{(t)} \geq 0$, a closed form solution of $Z^{(t)}$ could not be obtained. In the following, we introduce the Lagrangian multiplier $\gamma \in R^{n^{(t)} \times c^{(t)}}$, and the Lagrangian function is

$$
L\left(Z^{(t)}\right)=\left\|\phi\left(X^{(t)}\right)-M^{(t)} Z^{(t) T}\right\|_{F}^{2}-\operatorname{tr}\left(\gamma Z^{(t) T}\right) .
$$

Setting $\frac{\partial L\left(\mathbf{Z}^{t)}\right)}{\partial Z^{(t)}}=0$, we obtain $\gamma=-2 A+2 Z^{(t)} B$, where $A=\phi\left(X^{(t)}\right)^{T} M^{(t)}=F^{(t, t) \text { new }}$, $B=\left(M^{(t)}\right)^{T} M^{(t)}=G^{(t, t) \text { new }}$.

Using the Karush-Kuhn-Tucker condition $\gamma_{i j} Z_{i j}^{(t)}=0$ [Boyd and Vandenberghe 2004], we get $\left[-A+Z^{(t)} B\right]_{i j} Z_{i j}^{(t)}=0$. Introducing $A=A^{+}-A^{-}$and $B=B^{+}-B^{-}$, where $A_{i j}^{+}=\left(\left|A_{i j}\right|+A_{i j}\right) / 2$ and $A_{i j}^{-}=\left(\left|A_{i j}\right|-A_{i j}\right) / 2$ [Ding et al. 2010], we can obtain

$$
\left[A^{-}+Z^{(t)} B^{+}-A^{+}-Z^{(t)} B^{-}\right]_{i j} Z_{i j}^{(t)}=0 .
$$

Equation (24) leads to the following updating formula

$$
Z_{i j}^{(t)} \leftarrow Z_{i j}^{(t)} \sqrt{\frac{\left[A^{+}+Z^{(t)} B^{-}\right]_{i j}}{\left[A^{-}+Z^{(t)} B^{+}\right]_{i j}}} .
$$

Computation of $\boldsymbol{W}$ : Given $M$ and $Z$, each matrix $W^{t s}$ is independently determined by

$$
\begin{aligned}
& \min _{W^{t s}} \sum_{z=1}^{c^{(t)}} \sum_{l=1}^{c^{(s)}} w_{z l}^{t s}\left\|m_{z}^{(t)}-m_{l}^{(s)}\right\|_{2}^{2} \\
& \text { s.t. } \quad \sum_{z=1}^{c^{(t)}} w_{z l}^{t s}=\pi_{l}^{s}, \sum_{l=1}^{c^{(s)}} w_{z l}^{t s}=\pi_{z}^{t}, w_{z l}^{t s} \geq 0, \forall z, l .
\end{aligned}
$$


This problem can be efficiently solved by standard linear programming techniques. $\left\|m_{z}^{(t)}-m_{l}^{(s)}\right\|_{2}^{2}$ in Equation (26) can be calculated by

$$
\begin{aligned}
& \left\|m_{z}^{(t)}-m_{l}^{(s)}\right\|_{2}^{2} \\
& \quad=\left(m_{z}^{(t)}\right)^{T}\left(m_{z}^{(t)}\right)-2\left(m_{z}^{(t)}\right)^{T}\left(m_{l}^{(s)}\right)+\left(m_{l}^{(s)}\right)^{T}\left(m_{l}^{(s)}\right) \\
& \quad=G^{(t, t)}(z, z)-2 G^{(t, s)}(z, l)+G^{(s, s)}(l, l) .
\end{aligned}
$$

The local loss function in Equation (13) can be calculated by

$$
L^{(t)}\left(P^{(t)}, X^{(t)}\right)=\frac{1}{n^{(t)}} \operatorname{tr}\left(K^{(t, t)}-F^{(t, t)} Z^{(t) T}-Z^{(t)} F^{(t, t) T}+Z^{(t)} G^{(t, t)} Z^{(t) T}\right) .
$$

We present the optimization process of MKC in Algorithm 2.

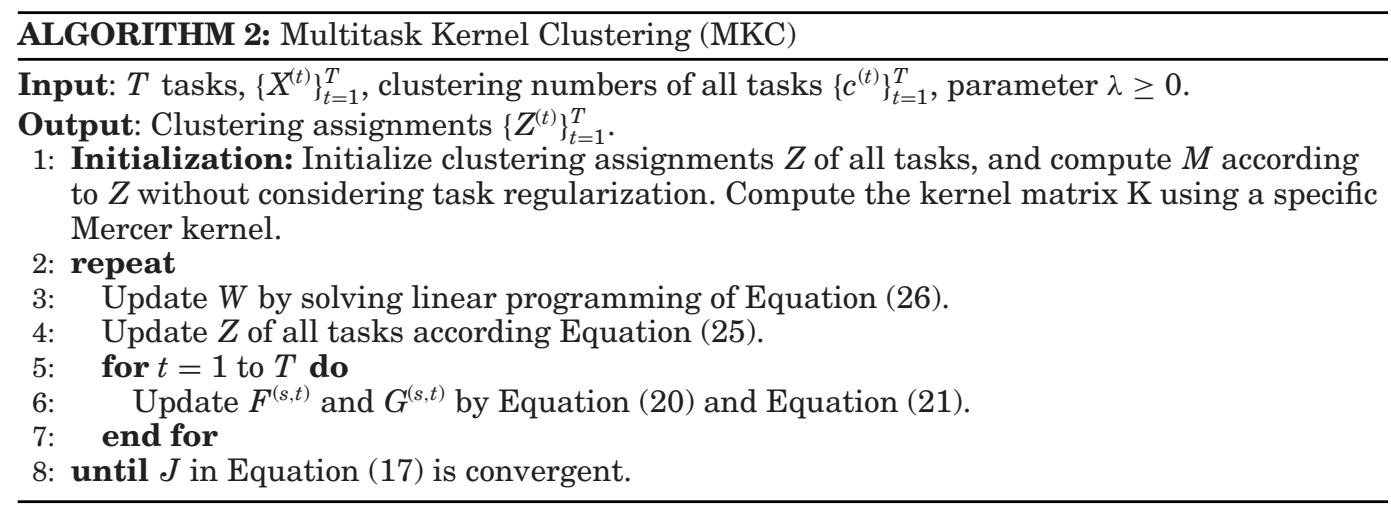

\subsection{Convergence Analysis of MKC}

Minimizing $J$ in Equation (16) is with respect to $W, Z$, and $M$. For $W$, fixing $Z$ and $M$, the computation of $W^{t s}$ in Equation (26) monotonically decreases the value of Equation (26) through the linear programming method [Zhang 1996]. For Z, fixing $W$ and $M$, the value of Equation (22) decreases monotonically under the updating rule for $Z^{(t)}$ in Equation (25) [Ding et al. 2010]. For $M$, fixing $W$ and $Z$, the Hessian matrix of $J_{1}$ in Equation (18) is $\frac{\partial^{2} J_{1}}{\partial M^{(t)} \partial\left(M^{(t)}\right)^{T}}=2\left(\frac{1}{n^{(t)}} Z^{(t) T} Z^{(t)}+2 \lambda E^{(t)}\right)$, which is positive semidefinite. Thus, the computation of $M^{(t)}$ in Equation (19) monotonically decreases the value of $J_{1}$. From the previous analysis, Algorithm 2 is guaranteed to converge.

\section{SMART MULTITASK KERNEL CLUSTERING}

\subsection{Avoiding the Negative Effects of MKC}

Suffering from similar issue as MBC, MKC can also make the centroids deviate from the relatively ideal positions which have already been found.

MKC gets the centroid matrix $M^{(t)}$ from Equation (19). If we set $\lambda=0$, we get $M^{(t)}=\phi\left(X^{(t)}\right) Z^{(t)}\left(Z^{(t) T} Z^{(t)}\right)^{-1}$, which is the centroid matrix calculated by KKM. In Equation (19), MKC utilizes the relationship $W^{s t}$ between the clusters of task $s$ and task $t$ to affect the centroid $M^{(t)}$ calculated by KKM. Therefore, minimizing the second term in Equation (16) has positive effects on the data points shared by other tasks to get their relatively ideal centroids, and negative effects on ones that are not shared by other tasks. 
A strategy to avoid the negative effects similar to that of S-MBC could be easily derived. We call the MKC algorithm with such a strategy S-MKC. The computations of $Z$ and $W$ in S-MKC are the same as MKC, thus we omit them here. For the computation of $M$ in S-MKC, there are two updating rules: (1) when the cluster centroids are obtained with the task regularization, optimizing $M$ is equivalent to optimizing $F$ in Equation (20) and $G$ in Equation (21); (2) when the cluster centroids are obtained without the task regularization, optimizing $M$ is equivalent to optimizing $F$ in Equation (20) and $G$ in Equation (21) with $\lambda=0$.

The overall optimization process of S-MKC is shown in Algorithm 3.

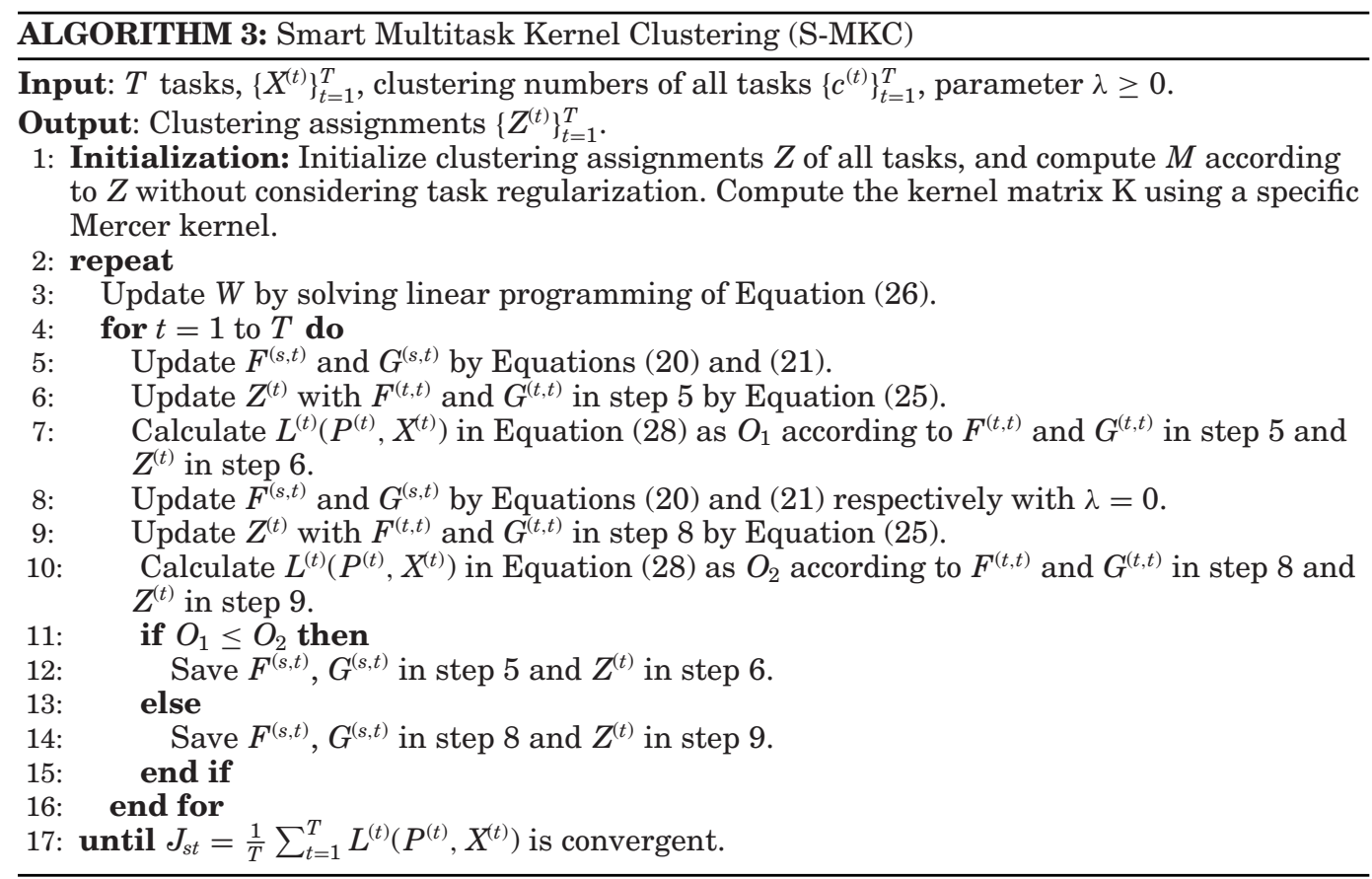

\subsection{Convergence Analysis of S-MKC}

Theorem 6.1. Algorithm 3 monotonically decreases the objective function $J_{s t}$.

Proof. Minimizing $J_{s t}$ is with respect to $Z$ and $M$. For $Z$, fixing $M$, the value of Equation (22) decreases monotonically under the updating rule for $Z^{(t)}$ in Equation (25) [Ding et al. 2010]. For $M$, there are two updating rules: (1) with task regularization, that is, under the updating rule for $M^{(t)}$ in Equation (19); (2) without task regularization, that is, $M^{(t)}=\phi\left(X^{(t)}\right) Z^{(t)}\left(Z^{(t) T} Z^{(t)}\right)^{-1}$.

Denote the objective value under iteration $i$ as $J_{s t}^{i}$, the objective value in the next iteration $i+1$ as

$$
J_{s t}^{i+1}=\left\{\begin{array}{l}
J_{s t}\left(M_{1 s t}^{(t)}, Z^{(t)}\right) \text { if } M^{(t)} \text { is updated by Eq. (19). } \\
J_{s t}\left(M_{2 n d}^{(t)}, Z^{(t)}\right) \text { otherwize. }
\end{array}\right.
$$


We first analyze the second rule. Fixing $Z$, the Hessian matrix of $\left\|\phi\left(X^{(t)}\right)-M^{(t)} Z^{(t) T}\right\|_{F}^{2}$ in $J_{s t}$ is $\frac{\partial^{2} J_{1}}{\partial M^{(t)} \partial\left(M^{(t)}\right)^{T}}=\frac{2}{n^{(t)}} Z^{(t) T} Z^{(t)}$, which is positive semi-definite. Thus, the computation of $M^{(t)}$ by the second rule monotonically decreases the value of $J_{s t}$, that is, $J_{s t}^{i+1}=$ $J_{s t}\left(M_{2 n d}^{(t)}, Z^{(t)}\right)<J_{s t}^{i}$.

We further prove that the computation of $M^{(t)}$ by the first rule can also monotonically decrease the value of $J_{s t}$. If $J_{s t}\left(M_{1 s t}^{(t)}, Z^{(t)}\right) \leq J_{s t}\left(M_{2 n d}^{(t)}, Z^{(t)}\right)$, then $J_{s t}^{i+1}=J_{s t}\left(M_{1 s t}^{(t)}, Z^{(t)}\right) \leq$ $J_{s t}\left(M_{2 n d}^{(t)}, Z^{(t)}\right)<J_{s t}^{i}$, otherwise $J_{s t}^{i+1}=J_{s t}\left(M_{2 n d}^{(t)}, Z^{(t)}\right)<J_{s t}^{i}$. Thus, the computation of $M^{(t)}$ by the first rule can also monotonically decrease the value of $J_{s t}$.

From the previous analysis, Algorithm 3 is guaranteed to converge.

\section{EXPERIMENTS}

We compare the proposed multitask clustering algorithms, S-MBC, MKC, and S-MKC, with typical single-task clustering algorithms, KM and KKM, typical multitask clustering algorithm MBC [Zhang and Zhang 2010], and the convex multitask clustering algorithm DMTRC which can deal with negative transfer problem [Zhang 2015]. We denote DMTRC implemented in the original space as DMTRC-L, and DMTRC implemented in the nonlinear feature mapping space with Gaussian kernel as DMTRC-R. All of our experiments have been performed on an Intel Core i5 3.2GHz Windows 7 machine with $8 \mathrm{~GB}$ memory.

\subsection{Evaluation Metrics}

We adopt two widely used metrics [Zhong and Ghosh 2003]: clustering accuracy (Acc) and normalized mutual information (NMI).

Acc measures the fraction of clustered instances that are relevant to the corresponding class.

$$
A c c=\frac{\sum_{i=1}^{n} \delta\left(\operatorname{map}\left(r_{i}\right), l_{i}\right)}{n},
$$

where $n$ denotes the total number of objects in a task, $r_{i}$ denotes the cluster label of $x_{i}, l_{i}$ denotes the true class label, $\delta(x, y)$ is the delta function that equals one if $x=y$, and equals zero otherwise, and $\operatorname{map}\left(r_{i}\right)$ is the permutation mapping function that maps each cluster label $r_{i}$ to the equivalent label from the dataset.

NMI is used for determining the quality of clusters. Given a clustering result, the NMI is calculated by

$$
N M I=\frac{\sum_{c_{i} \in C, c_{j}^{\prime} \in C^{\prime}} p\left(c_{i}, c_{j}^{\prime}\right) \cdot \log \frac{p\left(c_{i}, c_{j}^{\prime}\right)}{p\left(c_{i}\right) \cdot p\left(c_{j}^{\prime}\right)}}{\sqrt{\sum_{c_{i} \in C} p\left(c_{i}\right) \log p\left(c_{i}\right) \sum_{c_{j}^{\prime} \in C^{\prime}} p\left(c_{j}^{\prime}\right) \log p\left(c_{j}^{\prime}\right)}},
$$

where $p\left(c_{i}\right)$ and $p\left(c_{j}^{\prime}\right)$ denote the probabilities that an object arbitrarily selected from the dataset belongs to the clusters $c_{i}$ and $c_{j}^{\prime}$, respectively, and $p\left(c_{i}, c_{j}^{\prime}\right)$ is the joint probability that this arbitrarily selected object belongs to the clusters $c_{i}$ as well as $c_{j}^{\prime}$ at the same time.

\subsection{Datasets}

We use the 20Newsgroups dataset ${ }^{1}$ and some document datasets in $\mathrm{Xu}$ et al. [2003]. ${ }^{2}$

\footnotetext{
$\overline{{ }^{1} \mathrm{http}: / / q w o n e . c o m / j a s o n / 20 N e w s g r o u p s / . ~}$

${ }^{2}$ The clean data in matlab format are available form http://www.shi-zhong.com/software/docdata.zip.
} 
Table III. Datasets

\begin{tabular}{|c|c|c|c|c|}
\hline Dataset & Task id & \#Sample & \#Feature & Class \\
\hline \multirow{2}{*}{ NG1 } & Task 1 & 1561 & 3000 & 4 \\
\hline & Task 2 & 1560 & 3000 & 4 \\
\hline \multirow{2}{*}{ NG2 } & Task 1 & 1575 & 3000 & 4 \\
\hline & Task 2 & 1575 & 3000 & 2 \\
\hline \multirow[b]{2}{*}{ NG3 } & Task 1 & 1953 & 3000 & 5 \\
\hline & Task 2 & 1953 & 3000 & 5 \\
\hline \multirow{3}{*}{ Hitech } & Task 1 & 1030 & 10080 & $3(1-3)$ \\
\hline & Task 2 & 1148 & 10080 & $3(2-4)$ \\
\hline & Task 3 & 1700 & 10080 & $4(3-6)$ \\
\hline \multirow{2}{*}{ Ohscal } & Task 1 & 6118 & 11465 & $6(1-6)$ \\
\hline & Task 2 & 7529 & 11465 & $6(5-10)$ \\
\hline \multirow{3}{*}{ Reviews } & Task 1 & 3520 & 18482 & $3(1-3)$ \\
\hline & Task 2 & 2658 & 18482 & $3(2-4)$ \\
\hline & Task 3 & 1937 & 18482 & $3(3-5)$ \\
\hline \multirow{2}{*}{ Sports } & Task 1 & 4023 & 14870 & $4(2-5)$ \\
\hline & Task 2 & 3613 & 14870 & $4(4-7)$ \\
\hline \multirow{2}{*}{$\operatorname{Tr} 11$} & Task 1 & 256 & 6424 & $6(1-6)$ \\
\hline & Task 2 & 272 & 6424 & $6(4-9)$ \\
\hline \multirow{3}{*}{$\operatorname{Tr} 12$} & Task 1 & 102 & 5799 & $4(1-4)$ \\
\hline & Task 2 & 103 & 5799 & $4(3-6)$ \\
\hline & Task 3 & 241 & 5799 & $5(4-8)$ \\
\hline \multirow{2}{*}{$\operatorname{Tr} 23$} & Task 1 & 183 & 5831 & $4(1-4)$ \\
\hline & Task 2 & 157 & 5831 & $4(3-6)$ \\
\hline
\end{tabular}

The 20 Newsgroups is a collection of approximately 20000 newsgroup documents, partitioned across six root categories, under which are 20 subcategories. We use three splitting schemes to construct three datasets to demonstrate three typical cases of multitask clustering. For each dataset, we use the Rainbow toolkit for data processing. We remove the header lines and the stop words, and select the top 3,000 words by mutual information. The detailed constitutions of the three datasets are as follows.

(1) The first case is that all the datasets are from a same distribution. NG1 is constructed to represent this case, by splitting the selected documents into two parts, with each part having four sub categories: Comp.os.ms-windows.misc (class 3), Rec.motorcycles (class 9), Sci.electronics (class 13) and Talk.politics.mideast (class 18).

(2) The second case is that all the tasks are on an identical dataset but requires clusters at different resolutions. NG2 is constructed to represent this case. We select the documents in Comp.os.ms-windows.misc (class 3), Comp.sys.ibm.pc.hardware (class 4), Sci.crypt (class 12) and Sci.space (class 15), and combine the selected documents as the identical dataset, which also belong to Comp and Sci root categories.

(3) The third case is that the distributions of all the tasks are not identical but similar, the tasks share some data points from the same class labels. NG3 is constructed to represent this case. In NG3, task 1 is composed of the datasets from Comp.os.ms-win.misc (class 3), Comp.sys.ibm.pc.hardware (class 4), Rec.motocycle (class 9), Sci.electronics (class 13) and Talk.politics.mideast (class 18), task 2 is from Comp.sys.ibm.pc.hardware (class 4), Rec.sport.baseball (class 10), Sci.crypt (class 12), Sci.electronics (class 13) and Talk.politics.mideast (class 18).

The third case is the general case for multitask clustering, the remaining datasets which are from the CLUTO toolkit all belong to this case. We summarize the earlier datasets in Table III. The fifth column in Table III represents the number of clusters in each task, and the true class labels that the selected data belong to in the original dataset are in the brackets. 

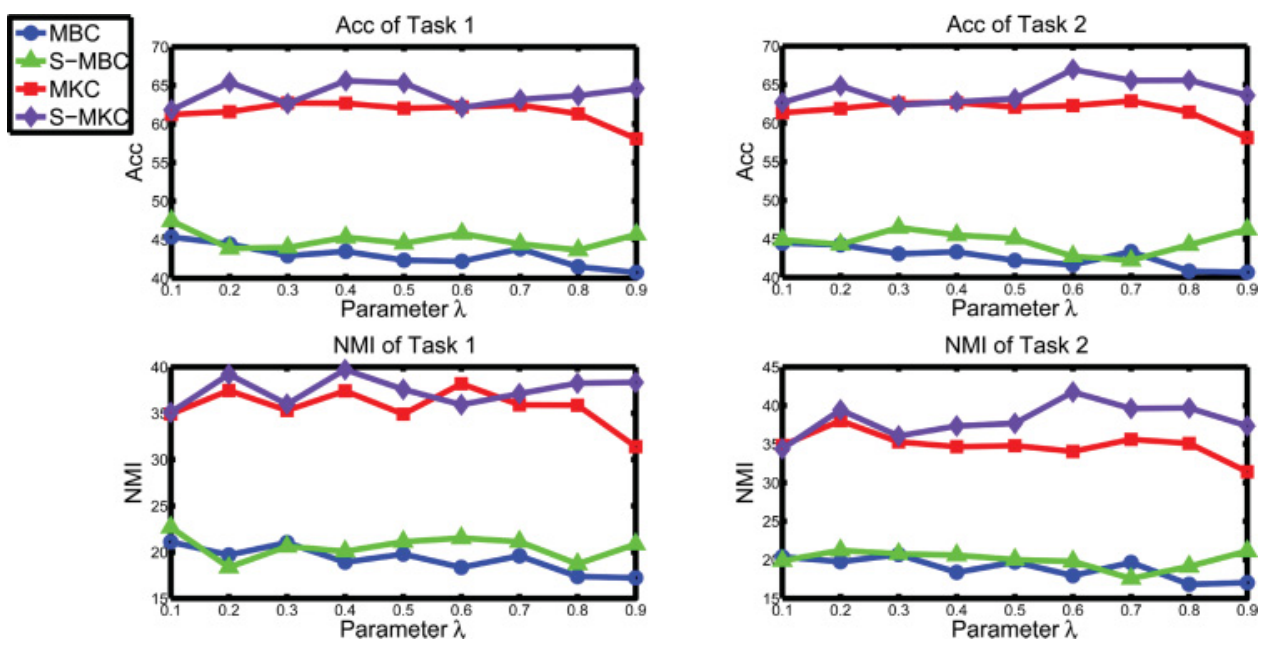

Fig. 2. The clustering results with different parameters on NG1.
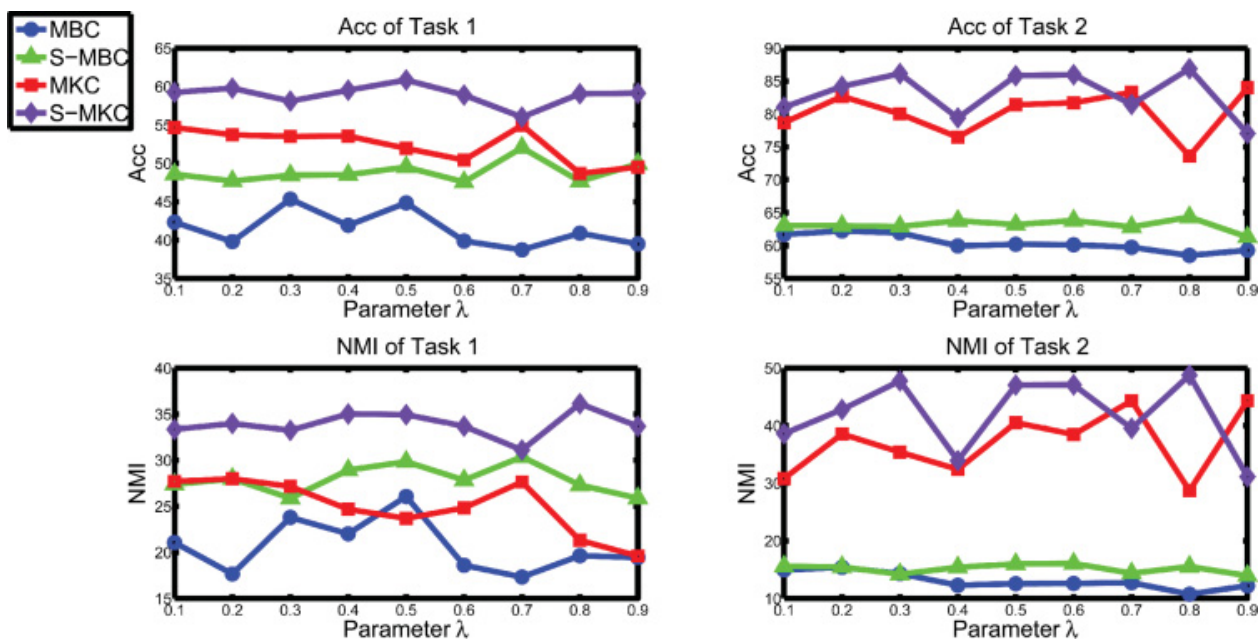

Fig. 3. The clustering results with different parameters on NG2.

\subsection{Parameter Investigation}

We set the number of clusters equal to the true number of classes for all the clustering algorithms. The Bregman divergence we choose is Euclidean distance. To tackle the nonlinear data clustering problem, we use the nonlinear Mercer kernel functionGaussian kernel function to compute the kernel matrix. The band width of the Gaussian kernel $\sigma$ is the median Euclidean distance between data points in the dataset. To investigate the impact of parameter $\lambda$ on the clustering performance, we perform MBC, S-MBC, MKC, and S-MKC methods by setting $\lambda$ equal to the value in $\{0.1,0.2,0.3$, $0.4,0.5,0.6,0.7,0.8,0.9\}$. We repeat each method 10 times, and the mean clustering results of the datasets NG1, NG2, and NG3 (representing the three cases) with different parameter settings are shown in Figure 2, Figure 3, and Figure 4. From Figure 2, Figure 3, and Figure 4, it can be seen that all the algorithms get fairly good performance with $\lambda=0.5$. Thus to make the results comparable, we set $\lambda=0.5$ for $\mathrm{MBC}$, $\mathrm{S}-\mathrm{MBC}, \mathrm{MKC}$, and S-MKC in all the datasets. Note that KM can be seen as a special 

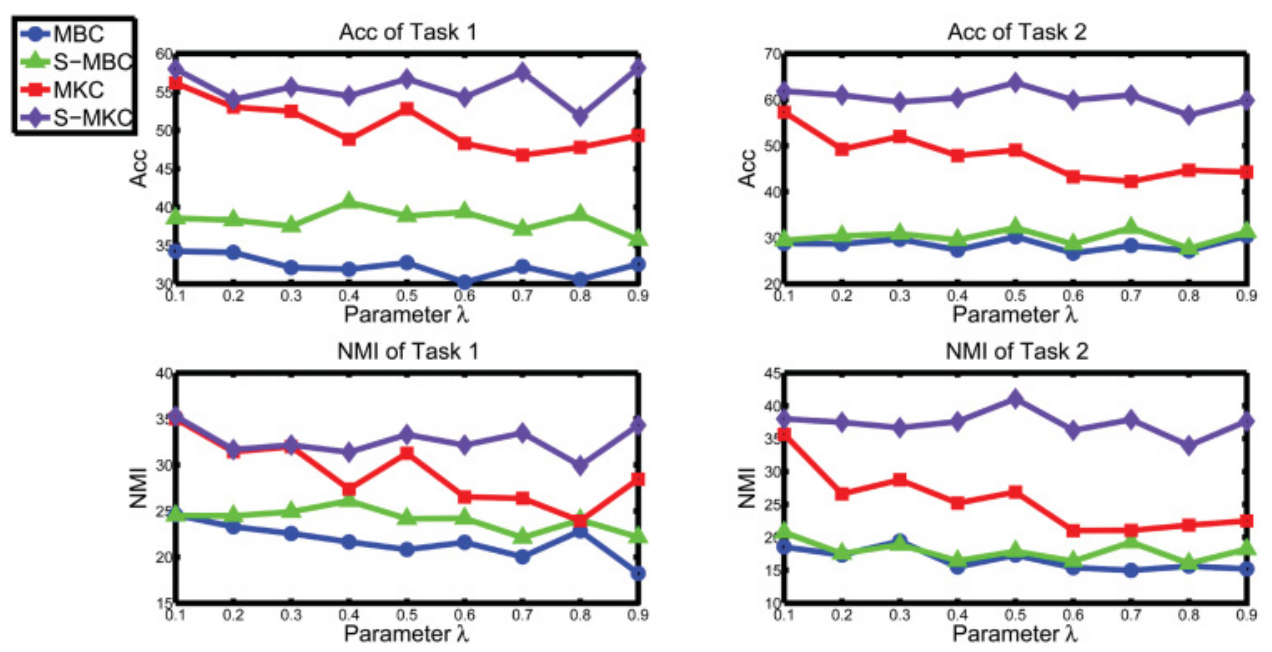

Fig. 4. The clustering results with different parameters on NG3.

case of the MBC algorithm using Euclidean distance with $\lambda=0$, and KKM can be seen as a special case of the MKC algorithm with $\lambda=0$.

For DMTRC-L and DMTRC-R, we apply the grid searching method to identify the parameters [Zhang 2015]. Specifically, $\lambda_{1}$ and $\lambda_{2}$ are both searched from $\left\{2^{-10}, 2^{-8}, \ldots, 2^{-2}\right\}$. For DMTRC-R, the Gaussian kernel width is the median Euclidean distance between data points in the dataset, which is the same as KKM, MKC, and S-MKC.

\subsection{Clustering Accuracy}

For KM, KKM, MBC, S-MBC, MKC, and S-MKC, we repeat each algorithm 10 times to compute the mean clustering results and the standard deviations on all the datasets in Table III. For DMTRC-L and DMTRC-R, since they are based on the assumption that all tasks have the same number of clusters, we only perform them on the datasets NG1, NG3, Ohscal, Reviews, Sports, Tr11 and Tr23 in Table III, which can meet such requirement. Moreover, as DMTRC-L and DMTRC-R are convex algorithms, we perform them one time under each parameter setting, and report the clustering performance corresponding to the best parameter setting. The results are shown in Table IV, Table V, $\ldots$, Table XIII.

7.4.1. $S-M B C$ and $S-M K C$ versus $M B C$. First, we summarise the following points from the clustering results of the algorithms KM, KKM, MBC, S-MBC, MKC, and S-MKC.

(1) When the tasks are from a same distribution and clustered into the same number of clusters (Case 1: Table IV), MBC and MKC perform better than their single task counterparts KM and KKM, respectively. This indicates that considering relationship among tasks does boost the clustering performance of related tasks. S-MBC and S-MKC improve on MBC and MKC a little, respectively. This is because that few negative effects of $\mathrm{MBC}$ and $\mathrm{MKC}$ occur in this kind of dataset.

(2) When the tasks are on an identical dataset but require clusters at different resolutions (Case2: Table V), MBC and MKC perform better than their single task counterparts KM and KKM respectively for Task 2, while perform worse for Task 1. This indicates that the clusters presented as containment are positively affected by the subclusters in the other tasks, while the clusters presented as subordination are negatively affected by the root-clusters in the other tasks, because they make the 
Table IV. Clustering Results on NG1

\begin{tabular}{|l|c|c|c|}
\hline Method & Task id & Acc(\%) & NMI(\%) \\
\hline \multirow{2}{*}{ KM } & Task 1 & $39.71 \pm 4.57$ & $18.82 \pm 8.29$ \\
\cline { 2 - 4 } & Task 2 & $40.73 \pm 5.98$ & $19.50 \pm 7.74$ \\
\hline \multirow{2}{*}{ MBC } & Task 1 & $42.32 \pm 4.91$ & $19.78 \pm 5.16$ \\
\cline { 2 - 4 } & Task 2 & $42.20 \pm 4.74$ & $19.70 \pm 5.13$ \\
\hline \multirow{2}{*}{ S-MBC } & Task 1 & $44.51 \pm 3.66$ & $21.12 \pm 4.21$ \\
\cline { 2 - 4 } & Task 2 & $45.03 \pm 4.88$ & $20.00 \pm 4.90$ \\
\hline \multirow{2}{*}{ DMTRC-L } & Task 1 & $\mathbf{9 6 . 6 0}$ & $\mathbf{8 7 . 7 1}$ \\
\cline { 2 - 4 } & Task 2 & $\mathbf{9 7 . 9 5}$ & $\mathbf{9 2 . 6 0}$ \\
\hline \multirow{2}{*}{ KKM } & Task 1 & $59.35 \pm 7.28$ & $31.93 \pm 6.20$ \\
\cline { 2 - 4 } & Task 2 & $60.43 \pm 6.13$ & $32.86 \pm 5.19$ \\
\hline \multirow{2}{*}{ MKC } & Task 1 & $62.03 \pm 5.26$ & $34.89 \pm 5.10$ \\
\cline { 2 - 4 } & Task 2 & $62.05 \pm 5.22$ & $34.79 \pm 4.88$ \\
\hline \multirow{2}{*}{ S-MKC } & Task 1 & $65.30 \pm 3.55$ & $37.57 \pm 5.78$ \\
\cline { 2 - 4 } & Task 2 & $63.16 \pm 5.41$ & $37.69 \pm 4.57$ \\
\hline \multirow{2}{*}{ DMTRC-R } & Task 1 & 93.15 & 79.87 \\
\cline { 2 - 4 } & Task 2 & 92.31 & 75.61 \\
\hline
\end{tabular}

Table V. Clustering Results on NG2

\begin{tabular}{|l|l|l|l|}
\hline Method & Task id & Acc(\%) & \multicolumn{1}{|c|}{ NMI $(\%)$} \\
\hline \multirow{2}{*}{ KM } & Task 1 & $47.68 \pm 4.77$ & $26.54 \pm 4.06$ \\
\cline { 2 - 4 } & Task 2 & $58.80 \pm 3.11$ & $12.04 \pm 4.05$ \\
\hline \multirow{2}{*}{ MBC } & Task 1 & $44.83 \pm 5.50$ & $26.06 \pm 5.92$ \\
\cline { 2 - 4 } & Task 2 & $60.19 \pm 2.88$ & $12.55 \pm 3.31$ \\
\hline \multirow{2}{*}{ S-MBC } & Task 1 & $49.50 \pm 2.90$ & $29.83 \pm 3.91$ \\
\cline { 2 - 4 } & Task 2 & $63.18 \pm 2.57$ & $15.96 \pm 3.08$ \\
\hline \multirow{2}{*}{ KKM } & Task 1 & $55.72 \pm 7.47$ & $28.82 \pm 4.58$ \\
\cline { 2 - 4 } & Task 2 & $75.63 \pm 9.72$ & $28.84 \pm 19.09$ \\
\hline \multirow{2}{*}{ MKC } & Task 1 & $51.94 \pm 4.85$ & $23.67 \pm 4.29$ \\
\cline { 2 - 4 } & Task 2 & $81.40 \pm 13.04$ & $40.52 \pm 21.58$ \\
\hline \multirow{2}{*}{ S-MKC } & Task 1 & $\mathbf{6 0 . 8 4} \pm \mathbf{6 . 8 2}$ & $\mathbf{3 4 . 9 3} \pm \mathbf{4 . 1 6}$ \\
\cline { 2 - 4 } & Task 2 & $\mathbf{8 5 . 7 9} \pm \mathbf{6 . 1 4}$ & $\mathbf{4 7 . 0 3} \pm \mathbf{1 4 . 2 9}$ \\
\hline
\end{tabular}

centroids of the clusters belonging to the same root cluster closer. S-MBC and S-MKC perform much better than MBC and MKC, respectively, since they employ strategies to identify and avoid the negative effects.

(3) When the distributions of all tasks are not identical but similar (Case 3: Table VI, Table VII, ..., Table XIII), compared with KM and KKM, MBC and MKC perform a little better in some datasets, but they perform worse in many other datasets. This is because that although $\mathrm{MBC}$ and MKC consider relationships among tasks, they both suffer from large amount of negative effects in this kind of datasets. However, S-MBC and S-MKC improve much on MBC and MKC, respectively, since they employ strategies to identify and avoid the negative effects.

(4) For near linear separable datasets Hitech, Ohscal, Reviews and Sports, Bregman clustering algorithms KM, MBC, and S-MBC perform better than their kernel counterparts KKM, MKC, and S-MKC, respectively, and S-MBC performs the best. This indicates that kernel algorithms by a nonlinear feature mapping do not help improve the clustering performance of near-linear separable data. Moreover, since an optimal bandwidth $\sigma$ of the Gaussian kernel is very hard to determine, kernel algorithms may perform worse than their original counterparts. 
Table VI. Clustering Results on NG3

\begin{tabular}{|c|c|c|c|}
\hline Method & Task id & $\operatorname{Acc}(\%)$ & $\operatorname{NMI}(\%)$ \\
\hline \multirow{2}{*}{$\mathrm{KM}$} & Task 1 & $33.64 \pm 3.51$ & $22.02 \pm 3.71$ \\
\hline & Task 2 & $28.01 \pm 3.09$ & $16.31 \pm 4.80$ \\
\hline \multirow{2}{*}{$\mathrm{MBC}$} & Task 1 & $32.75 \pm 4.30$ & $20.81 \pm 6.42$ \\
\hline & Task 2 & $30.24 \pm 4.58$ & $17.30 \pm 5.97$ \\
\hline \multirow{2}{*}{ S-MBC } & Task 1 & $38.84 \pm 4.48$ & $24.15 \pm 4.61$ \\
\hline & Task 2 & $32.18 \pm 2.04$ & $17.85 \pm 5.28$ \\
\hline \multirow{2}{*}{ DMTRC-L } & Task 1 & 87.61 & 68.69 \\
\hline & Task 2 & 84.84 & 62.31 \\
\hline \multirow{2}{*}{ KKM } & Task 1 & $49.14 \pm 7.81$ & $25.80 \pm 6.46$ \\
\hline & Task 2 & $56.77 \pm 7.03$ & $33.41 \pm 6.42$ \\
\hline \multirow{2}{*}{ MKC } & Task 1 & $52.80 \pm 3.14$ & $31.25 \pm 4.00$ \\
\hline & Task 2 & $49.03 \pm 3.90$ & $26.87 \pm 3.79$ \\
\hline \multirow{2}{*}{ S-MKC } & Task 1 & $56.69 \pm 5.00$ & $33.29 \pm 4.18$ \\
\hline & Task 2 & $63.70 \pm 5.34$ & $41.05 \pm 3.78$ \\
\hline \multirow{2}{*}{ DMTRC-R } & Task 1 & 83.82 & 65.08 \\
\hline & Task 2 & 81.26 & 58.65 \\
\hline
\end{tabular}

Table VII. Clustering Results on Hitech

\begin{tabular}{|l|c|c|c|}
\hline Method & Task id & Acc(\%) & NMI $(\%)$ \\
\hline \multirow{3}{*}{ KM } & Task 1 & $66.36 \pm 5.91$ & $38.30 \pm 8.63$ \\
\cline { 2 - 4 } & Task 2 & $43.49 \pm 2.41$ & $8.54 \pm 2.45$ \\
\cline { 2 - 4 } & Task 3 & $42.80 \pm 3.72$ & $14.17 \pm 2.45$ \\
\hline \multirow{3}{*}{ MBC } & Task 1 & $60.79 \pm 10.55$ & $27.22 \pm 11.62$ \\
\cline { 2 - 4 } & Task 2 & $43.70 \pm 2.86$ & $5.87 \pm 2.63$ \\
\cline { 2 - 4 } & Task 3 & $33.80 \pm 2.82$ & $3.58 \pm 1.96$ \\
\hline \multirow{3}{*}{ S-MBC } & Task 1 & $\mathbf{7 2 . 3 6} \pm \mathbf{5 . 4 7}$ & $\mathbf{4 5 . 2 6} \pm \mathbf{6 . 6 1}$ \\
\cline { 2 - 4 } & Task 2 & $\mathbf{4 6 . 9 3} \pm \mathbf{2 . 7 7}$ & $\mathbf{9 . 2 9} \pm \mathbf{3 . 1 4}$ \\
\cline { 2 - 4 } & Task 3 & $\mathbf{4 4 . 4 3} \pm \mathbf{3 . 6 0}$ & $\mathbf{1 5 . 1 0} \pm \mathbf{2 . 9 7}$ \\
\hline \multirow{3}{*}{ MKM } & Task 1 & $66.15 \pm 5.05$ & $37.13 \pm 6.80$ \\
\cline { 2 - 4 } & Task 2 & $39.73 \pm 3.28$ & $5.00 \pm 2.43$ \\
\cline { 2 - 4 } & Task 3 & $38.22 \pm 3.54$ & $9.29 \pm 2.35$ \\
\hline \multirow{3}{*}{ S-MKC } & Task 1 & $42.90 \pm 4.95$ & $1.90 \pm 2.14$ \\
\cline { 2 - 4 } & Task 2 & $41.75 \pm 6.01$ & $1.18 \pm 0.79$ \\
\cline { 2 - 4 } & Task 3 & $31.69 \pm 3.30$ & $0.82 \pm 0.57$ \\
\cline { 2 - 4 } & Task 1 & $68.21 \pm 3.76$ & $37.18 \pm 7.55$ \\
\cline { 2 - 4 } & Task 2 & $45.74 \pm 2.65$ & $8.57 \pm 1.87$ \\
\cline { 2 - 4 } & Task 3 & $40.74 \pm 3.71$ & $10.40 \pm 1.58$ \\
\hline
\end{tabular}

(5) For nonlinear separable datasets such as NG1, NG2, NG3, Tr11, Tr12 and Tr23, the kernel algorithms KKM, MKC, and S-MKC perform better than their original counterparts KM, MBC, and S-MBC, respectively, and S-MKC performs the best. This shows that S-MKC can deal with multitask clustering of nonlinear separable data well.

7.4.2. S-MBC and S-MKC versus DMTRC-L and DMTRC-R. Secondly, we summarise the following points from the clustering results of the algorithms S-MBC, S-MKC, DMTRCL, and DMTRC-R.

(1) For near linear separable datasets Ohscal, Reviews and Sports, the DMTRC algorithm implemented in the original space DMTRC-L performs better than the DMTRC algorithm implemented in the nonlinear feature mapping space DMTRC-R. 
Table VIII. Clustering Results on Ohscal

\begin{tabular}{|l|c|c|c|}
\hline Method & Task id & Acc(\%) & NMI(\%) \\
\hline \multirow{2}{*}{ KM } & Task 1 & $43.51 \pm 5.88$ & $23.45 \pm 2.88$ \\
\cline { 2 - 4 } & Task 2 & $39.26 \pm 2.44$ & $25.80 \pm 3.41$ \\
\hline \multirow{2}{*}{ MBC } & Task 1 & $43.56 \pm 4.75$ & $21.83 \pm 2.48$ \\
\cline { 2 - 4 } & Task 2 & $40.38 \pm 2.72$ & $28.39 \pm 2.68$ \\
\hline \multirow{2}{*}{ S-MBC } & Task 1 & $48.32 \pm 5.09$ & $25.07 \pm 2.73$ \\
\cline { 2 - 4 } & Task 2 & $42.94 \pm 2.33$ & $30.52 \pm 2.34$ \\
\hline \multirow{2}{*}{ DMTRC-L } & Task 1 & $\mathbf{7 0 . 5 1}$ & $\mathbf{5 3 . 0 5}$ \\
\cline { 2 - 4 } & Task 2 & $\mathbf{8 4 . 8 7}$ & $\mathbf{6 5 . 4 4}$ \\
\hline \multirow{2}{*}{ KKM } & Task 1 & $38.85 \pm 3.42$ & $18.21 \pm 1.90$ \\
\cline { 2 - 4 } & Task 2 & $39.54 \pm 4.39$ & $21.68 \pm 2.97$ \\
\hline \multirow{2}{*}{ MKC } & Task 1 & $42.71 \pm 2.88$ & $22.39 \pm 1.52$ \\
\cline { 2 - 4 } & Task 2 & $39.89 \pm 2.83$ & $24.85 \pm 1.63$ \\
\hline \multirow{2}{*}{ S-MKC } & Task 1 & $47.69 \pm 2.86$ & $24.38 \pm 3.59$ \\
\cline { 2 - 4 } & Task 2 & $42.61 \pm 3.29$ & $25.68 \pm 3.24$ \\
\hline \multirow{2}{*}{ DMTRC-R } & Task 1 & 70.25 & 51.65 \\
\cline { 2 - 4 } & Task 2 & 82.71 & 61.21 \\
\hline
\end{tabular}

Table IX. Clustering Results on Reviews

\begin{tabular}{|l|l|l|c|}
\hline Method & Task id & \multicolumn{1}{|c|}{ Acc(\%) } & NMI $(\%)$ \\
\hline \multirow{3}{*}{ KM } & Task 1 & $66.36 \pm 6.74$ & $38.89 \pm 5.99$ \\
\cline { 2 - 4 } & Task 2 & $67.65 \pm 10.34$ & $38.09 \pm 9.44$ \\
\cline { 2 - 4 } & Task 3 & $68.25 \pm 11.85$ & $28.17 \pm 12.08$ \\
\hline \multirow{3}{*}{ MBC } & Task 1 & $48.01 \pm 7.78$ & $15.72 \pm 13.69$ \\
\cline { 2 - 4 } & Task 2 & $53.29 \pm 10.51$ & $36.46 \pm 10.02$ \\
\cline { 2 - 4 } S-MBC & Task 3 & $71.55 \pm 9.33$ & $21.10 \pm 9.79$ \\
\hline \multirow{3}{*}{ DMTRC-L } & Task 1 & $69.46 \pm 6.25$ & $41.01 \pm 6.36$ \\
\cline { 2 - 4 } & Task 2 & $\mathbf{7 6 . 3 9} \pm \mathbf{6 . 9 6}$ & $\mathbf{4 2 . 9 7} \pm \mathbf{8 . 1 7}$ \\
\cline { 2 - 4 } & Task 3 & $\mathbf{7 5 . 2 0} \pm \mathbf{1 0 . 9 6}$ & $31.20 \pm 14.87$ \\
\cline { 2 - 4 } & Task 1 & $\mathbf{8 8 . 5 8}$ & $\mathbf{6 7 . 4 8}$ \\
\cline { 2 - 4 } & Task 2 & 47.71 & 9.37 \\
\hline \multirow{3}{*}{ KKM } & Task 3 & 52.56 & $\mathbf{3 6 . 6 6}$ \\
\cline { 2 - 4 } & Task 1 & $60.59 \pm 9.00$ & $31.01 \pm 6.23$ \\
\cline { 2 - 4 } & Task 2 & $62.87 \pm 9.06$ & $27.97 \pm 8.94$ \\
\hline \multirow{3}{*}{ MKC } & Task 3 & $61.93 \pm 1.92$ & $32.24 \pm 2.25$ \\
\cline { 2 - 4 } & Task 1 & $48.11 \pm 6.25$ & $9.12 \pm 6.05$ \\
\cline { 2 - 4 } & Task 2 & $50.30 \pm 5.03$ & $8.32 \pm 6.62$ \\
\cline { 2 - 4 } S-MKC & Task 3 & $49.13 \pm 9.90$ & $8.04 \pm 5.39$ \\
\cline { 2 - 4 } & Task 1 & $68.83 \pm 10.15$ & $39.36 \pm 8.91$ \\
\cline { 2 - 4 } & Task 2 & $68.62 \pm 13.80$ & $32.86 \pm 14.04$ \\
\cline { 2 - 4 } & Task 3 & $64.52 \pm 0.71$ & $36.53 \pm 1.93$ \\
\hline \multirow{2}{*}{ DMTRC-R } & Task 1 & 70.31 & 27.72 \\
\cline { 2 - 4 } & Task 2 & 54.59 & 13.58 \\
\cline { 2 - 4 } & Task 3 & 51.68 & 15.68 \\
\hline
\end{tabular}

(2) For nonlinear separable datasets such as Tr11 and Tr23, DMTRC-R performs better than DMTRC-L. For datasets NG1 and NG3, DMTRC-L performs better than DMTRC-R. This is because the Gaussian kernel width DMTRC-R used in the experiments may not be optimal. However, it is another issue to find the optimal Gaussian kernel width for DMTRC-R on every dataset, which is hard to solve and is beyond the scope of this article. 
Table X. Clustering Results on Sports

\begin{tabular}{|l|c|c|c|}
\hline Method & Task id & Acc $(\%)$ & NMI $(\%)$ \\
\hline \multirow{2}{*}{ KM } & Task 1 & $53.70 \pm 11.85$ & $28.00 \pm 11.53$ \\
\cline { 2 - 4 } & Task 2 & $53.27 \pm 7.68$ & $33.02 \pm 8.98$ \\
\hline \multirow{2}{*}{ MBC } & Task 1 & $54.89 \pm 5.29$ & $23.40 \pm 5.01$ \\
\cline { 2 - 4 } & Task 2 & $50.01 \pm 6.07$ & $24.59 \pm 5.99$ \\
\hline \multirow{2}{*}{ S-MBC } & Task 1 & $\mathbf{5 6 . 1 5} \pm \mathbf{8 . 8 7}$ & $32.88 \pm 7.53$ \\
\cline { 2 - 4 } & Task 2 & $\mathbf{5 5 . 3 0} \pm \mathbf{7 . 6 4}$ & $35.24 \pm 8.11$ \\
\hline \multirow{2}{*}{ DMTRC-L } & Task 1 & 56.08 & $\mathbf{4 4 . 4 3}$ \\
\cline { 2 - 4 } & Task 2 & 54.58 & $\mathbf{3 9 . 4 1}$ \\
\hline \multirow{2}{*}{ KKM } & Task 1 & $45.50 \pm 3.00$ & $24.97 \pm 6.47$ \\
\cline { 2 - 4 } & Task 2 & $50.45 \pm 3.02$ & $33.06 \pm 4.17$ \\
\hline \multirow{2}{*}{ MKC } & Task 1 & $48.21 \pm 2.72$ & $25.95 \pm 4.47$ \\
\cline { 2 - 4 } & Task 2 & $49.43 \pm 3.34$ & $27.44 \pm 5.23$ \\
\hline \multirow{2}{*}{ S-MKC } & Task 1 & $48.79 \pm 3.54$ & $26.98 \pm 4.13$ \\
\cline { 2 - 4 } & Task 2 & $51.83 \pm 2.21$ & $35.11 \pm 3.06$ \\
\hline \multirow{2}{*}{ DMTRC-R } & Task 1 & 48.70 & 18.64 \\
\cline { 2 - 4 } & Task 2 & 51.23 & 20.56 \\
\hline
\end{tabular}

(3) For datasets NG2, Hitech, and Tr12 in which the tasks have different numbers of clusters, the positive and negative task correlations learning in DMTRC-L and DMTRC-R cannot be performed, since it requires the number of clusters in different tasks to be identical. However, S-MBC and S-MKC work well on these datasets, this advantage is inherited from MBC.

(4) For datasets NG1, NG3 and Ohscal, DMTRC-L, and DMTRC-R perform much better than S-MBC and S-MKC, this is because that these datasets satisfy the assumption of DMTRC, that is, the empirical label marginal distribution in each task distributes evenly. Moreover, since the DMTRC methods use convex optimization, they can get the global optimal solution.

(5) For dataset Tr11, S-MBC and S-MKC perform better than DMTRC-L and DMTRC-R, this is because that Tr11 does not satisfy the assumption of DMTRC, that is, the empirical label marginal distribution in each task does not distribute evenly.

(6) For datasets Reviews, Sports and Tr23, our proposed algorithms (S-MBC and SMKC) and the DMTRC algorithms (DMTRC-L and DMTRC-R) alternatively perform better on different tasks or under different evaluation metrics. This is because that some tasks in these datasets satisfy or approach the assumption of DMTRC, whereas the other tasks do not. For example, it can be observed that in the dataset Reviews, the empirical label marginal distribution of Task 1 distributes very evenly, whereas the empirical label marginal distribution of Task 2 and Task 3 distributes very unevenly.

\subsection{Computational Time}

In this subsection, we investigate the computational time of MBC [Zhang and Zhang 2010], S-MBC, MKC, S-MKC, DMTRC-L, and DMTRC-R [Zhang 2015]. The parameters are the same as those in the clustering performance subsection. Note that DMTRC-L and DMTRC-R are based on the assumption that all tasks have the same number of clusters, so we only report the computational time of them on the datasets NG1, NG3, Ohscal, Reviews, Sports, Tr11 and Tr23.

From Figure 5, it can be seen that MBC is the fastest algorithm, DMTRC-L and DMTRC-R are the two slowest algorithms.

The computational time of S-MBC and S-MKC is a little lower than twice the computational time of $\mathrm{MBC}$ and $\mathrm{MKC}$, respectively. The computational time of multitask kernel algorithms MKC and S-MKC is basically one order of magnitude higher than 
Table XI. Clustering Results on $\operatorname{Tr} 11$

\begin{tabular}{|l|c|c|c|}
\hline Method & Task id & Acc $(\%)$ & NMI $(\%)$ \\
\hline \multirow{2}{*}{ KM } & Task 1 & $31.36 \pm 5.09$ & $11.81 \pm 6.44$ \\
\cline { 2 - 4 } & Task 2 & $35.47 \pm 3.58$ & $3.27 \pm 1.10$ \\
\hline \multirow{2}{*}{ MBC } & Task 1 & $37.89 \pm 5.33$ & $15.45 \pm 6.81$ \\
\cline { 2 - 4 } & Task 2 & $44.85 \pm 3.63$ & $7.40 \pm 2.34$ \\
\hline \multirow{2}{*}{ S-MBC } & Task 1 & $44.02 \pm 8.00$ & $29.10 \pm 9.04$ \\
\cline { 2 - 4 } & Task 2 & $46.61 \pm 0.96$ & $8.79 \pm 4.63$ \\
\hline \multirow{2}{*}{ DMTRC-L } & Task 1 & 43.53 & 22.20 \\
\cline { 2 - 4 } & Task 2 & 48.16 & 42.70 \\
\hline \multirow{2}{*}{ KKM } & Task 1 & $49.96 \pm 6.44$ & $42.21 \pm 5.98$ \\
\cline { 2 - 4 } & Task 2 & $44.77 \pm 6.92$ & $37.65 \pm 7.27$ \\
\hline \multirow{2}{*}{ MKC } & Task 1 & $50.89 \pm 4.48$ & $39.50 \pm 5.80$ \\
\cline { 2 - 4 } & Task 2 & $54.15 \pm 8.08$ & $43.14 \pm 6.58$ \\
\hline \multirow{2}{*}{ S-MKC } & Task 1 & $\mathbf{5 4 . 8 4} \pm \mathbf{5 . 4 6}$ & $\mathbf{4 7 . 6 6} \pm \mathbf{5 . 4 3}$ \\
\cline { 2 - 4 } & Task 2 & $\mathbf{5 5 . 3 9} \pm \mathbf{4 . 6 4}$ & $\mathbf{4 4 . 2 9} \pm \mathbf{6 . 2 2}$ \\
\hline \multirow{2}{*}{ DMTRC-R } & Task 1 & 46.88 & 24.46 \\
\cline { 2 - 4 } & Task 2 & 51.47 & 30.22 \\
\hline
\end{tabular}

Table XII. Clustering Results on Tr12

\begin{tabular}{|l|l|l|c|}
\hline Method & Task id & Acc $(\%)$ & NMI $(\%)$ \\
\hline \multirow{3}{*}{ KM } & Task 1 & $35.68 \pm 2.93$ & $7.05 \pm 5.45$ \\
\cline { 2 - 4 } & Task 2 & $34.75 \pm 2.03$ & $4.94 \pm 2.02$ \\
\cline { 2 - 4 } & Task 3 & $33.02 \pm 2.32$ & $3.64 \pm 0.93$ \\
\hline \multirow{3}{*}{ MBC } & Task 1 & $35.19 \pm 3.11$ & $3.55 \pm 1.69$ \\
\cline { 2 - 4 } & Task 2 & $35.92 \pm 2.54$ & $3.41 \pm 2.21$ \\
\cline { 2 - 4 } & Task 3 & $27.55 \pm 1.71$ & $2.54 \pm 0.64$ \\
\hline \multirow{3}{*}{ S-MBC } & Task 1 & $36.86 \pm 3.03$ & $6.40 \pm 4.44$ \\
\cline { 2 - 4 } & Task 2 & $36.40 \pm 2.10$ & $6.30 \pm 3.73$ \\
\cline { 2 - 4 } & Task 3 & $34.32 \pm 2.10$ & $4.78 \pm 0.81$ \\
\hline \multirow{3}{*}{ KKM } & Task 1 & $67.94 \pm 9.10$ & $55.40 \pm 11.91$ \\
\cline { 2 - 4 } & Task 2 & $63.89 \pm 10.85$ & $43.14 \pm 11.06$ \\
\cline { 2 - 4 } & Task 3 & $48.09 \pm 6.31$ & $29.80 \pm 5.92$ \\
\hline \multirow{3}{*}{ MKC } & Task 1 & $37.15 \pm 3.82$ & $4.89 \pm 3.18$ \\
\cline { 2 - 4 } & Task 2 & $35.14 \pm 3.16$ & $4.07 \pm 2.08$ \\
\cline { 2 - 4 } & Task 3 & $30.62 \pm 2.76$ & $3.68 \pm 1.57$ \\
\hline \multirow{2}{*}{ S-MKC } & Task 1 & $\mathbf{7 2 . 9 4} \pm \mathbf{8 . 0 7}$ & $\mathbf{5 9 . 3 0} \pm \mathbf{7 . 1 7}$ \\
\cline { 2 - 4 } & Task 2 & $\mathbf{6 7 . 1 8} \pm \mathbf{7 . 1 6}$ & $\mathbf{4 8 . 5 6} \pm \mathbf{6 . 2 8}$ \\
\cline { 2 - 4 } & Task 3 & $\mathbf{5 2 . 4 8} \pm \mathbf{6 . 9 4}$ & $\mathbf{3 5 . 3 9} \pm \mathbf{7 . 6 1}$ \\
\hline
\end{tabular}

multitask Bregman algorithms MBC and S-MBC, because MKC and S-MKC need additional steps to compute the Gaussian kernel. The computational time of DMTRC-R is one or two orders of magnitude higher than that of DMTRC-L, because DMTRC-R spends extra time computing the Gaussian kernel matrix of the selected training data and the testing data.

Comparing the two algorithms implemented in the original space, that is, DMTRC-L and S-MBC, the computational time of DMTRC-L is one order of magnitude higher than that of S-MBC on the datasets such as NG1, Reviews, Sports, Tr11 and Tr23, and two orders of magnitude higher than that of S-MBC on the datasets such as NG3 and Ohscal. 
Table XIII. Clustering Results on Tr23

\begin{tabular}{|l|c|c|c|}
\hline Method & Task id & Acc(\%) & NMI $(\%)$ \\
\hline \multirow{2}{*}{ KM } & Task 1 & $40.20 \pm 0.78$ & $5.74 \pm 0.96$ \\
\cline { 2 - 4 } & Task 2 & $47.70 \pm 2.88$ & $7.00 \pm 1.24$ \\
\hline \multirow{2}{*}{ MBC } & Task 1 & $41.09 \pm 2.06$ & $6.31 \pm 2.28$ \\
\cline { 2 - 4 } & Task 2 & $47.40 \pm 2.28$ & $5.87 \pm 1.97$ \\
\hline \multirow{2}{*}{ S-MBC } & Task 1 & $41.53 \pm 2.13$ & $7.31 \pm 1.55$ \\
\cline { 2 - 4 } & Task 2 & $48.59 \pm 1.20$ & $7.29 \pm 1.69$ \\
\hline \multirow{2}{*}{ DMTRC-L } & Task 1 & 52.46 & 29.61 \\
\cline { 2 - 4 } & Task 2 & 43.31 & 20.61 \\
\hline \multirow{2}{*}{ KKM } & Task 1 & $49.12 \pm 5.67$ & $21.04 \pm 4.78$ \\
\cline { 2 - 4 } & Task 2 & $48.02 \pm 3.83$ & $29.94 \pm 4.47$ \\
\hline \multirow{2}{*}{ MKC } & Task 1 & $47.86 \pm 4.80$ & $21.31 \pm 4.41$ \\
\cline { 2 - 4 } & Task 2 & $51.97 \pm 4.60$ & $31.56 \pm 4.89$ \\
\hline \multirow{2}{*}{ S-MKC } & Task 1 & $51.31 \pm 4.58$ & $22.80 \pm 3.39$ \\
\cline { 2 - 4 } & Task 2 & $\mathbf{5 2 . 9 9} \pm \mathbf{4 . 8 6}$ & $\mathbf{3 5 . 8 2} \pm \mathbf{6 . 0 2}$ \\
\hline \multirow{2}{*}{ DMTRC-R } & Task 1 & $\mathbf{6 1 . 7 5}$ & $\mathbf{3 0 . 9 3}$ \\
\cline { 2 - 4 } & Task 2 & 40.76 & 16.94 \\
\hline
\end{tabular}

$\square \mathrm{MBC} \square$ S-MBC $\square$ MKC $\square$ S-MKC $\square$ DMTRC-L $\square$ DMTRC-R

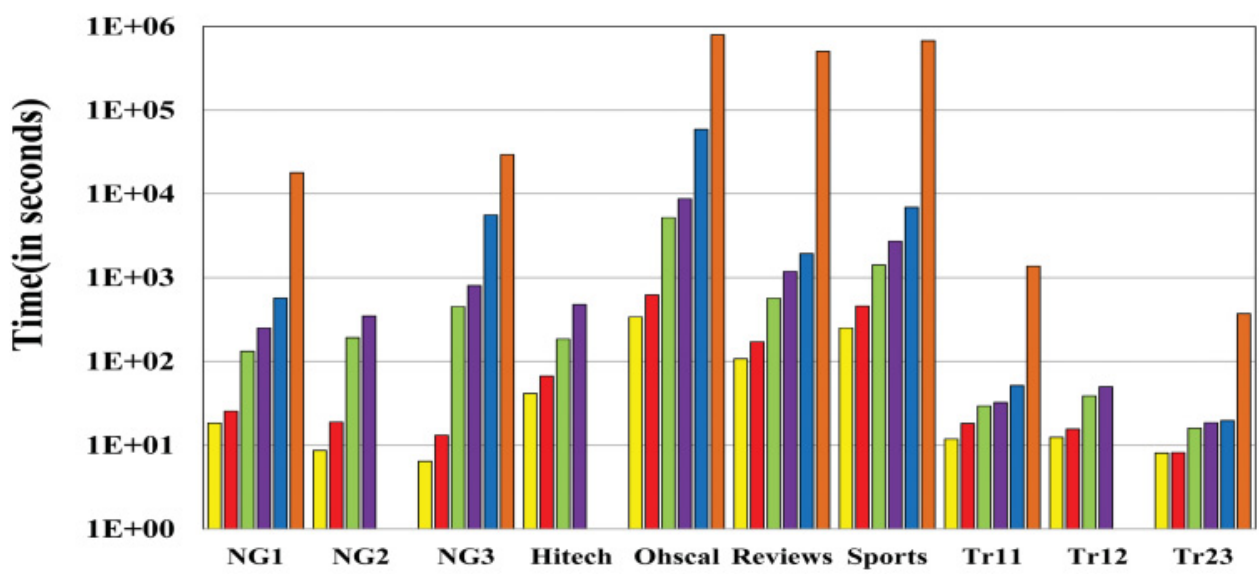

Data Sets

Fig. 5. The computational time of MBC, S-MBC, MKC, S-MKC, DMTRC-L, and DMTRC-R on the datasets in Table III. As DMTRC-L and DMTRC-R are based on the assumption that all tasks have the same number of clusters, we only report the computational time of them on the datasets NG1, NG3, Ohscal, Reviews, Sports, Tr11 and Tr23.

Comparing the two algorithms implemented in the nonlinear feature mapping space, that is, DMTRC-R and S-MKC, the computational time of DMTRC-R is one or two orders of magnitude higher than that of S-MKC.

\subsection{Summary}

Compared to MBC, S-MBC achieves better clustering accuracy, and its computational time is no more than twice of that of MBC. MKC and S-MKC achieve better clustering accuracy than MBC for nonlinear separable data, with computational time one order of magnitude higher than that of MBC. 
Compared to DMTRC-L and DMTRC-R, S-MBC, and S-MKC perform worse in terms of clustering accuracy in some special cases, but better or comparable in other cases. Moreover, S-MBC and S-MKC can work well in datasets with different cluster numbers among different tasks, whereas DMTRC-L and DMTRC-R do not work. DMTRC-L and DMTRC-R consume one or two orders of magnitude higher computational time than S-MBC and S-MKC.

Synthetically considering accuracy, efficiency, and applicability, our proposed algorithms S-MBC and S-MKC are competitive compared to the MBC, DMTRC-L, and DMTRC-R algorithms.

\section{RELATED WORK}

\subsection{Multitask Learning}

Multitask learning [Caruana 1997] has gained a lot of attention in the past decade due to its good predictive performance. There are two main approaches to learn the relationship among related tasks: sharing a common feature representation and sharing common model parameters. Typical methods of sharing a common feature representation are to share common underlying structures [Chen et al. 2009; Ando and Zhang 2005; Argyriou et al. 2006]. Typical methods of sharing common model parameters include using the common prior distribution in hierarchical Bayesian models [Bakker and Heskes 2003], exploiting kernel-based methods with regularization [Evgeniou and Pontil 2004; Evgeniou et al. 2005; Micchelli and Pontil 2004], sharing the parameters of Gaussian process [Bonilla et al. 2007; Lawrence and Platt 2004]. However, most of multitask learning methods are supervised.

Since there are many clustering tasks which are closely related in the real world, recently multitask clustering (unsupervised multitask learning) has become a hot topic. There are various ways to learn the relationships among different tasks from unlabeled data. Gu and Zhou [2009] learned a subspace shared by multiple related tasks. Gu et al. [2011] handled multitask clustering problem by learning a kernel. Nguyen et al. [2011, 2013] proposed a feature free and parameter light multitask clustering framework based on Kolmogorov complexity. Xie et al. [2012] proposed a multitask co-clustering method by learning the relationships of features among different tasks. Zhang and Zhou [2012] learned a shared subspace through domain adaptation. All of the methods earlier focus on cross-domain multitask clustering, which deals with an ideal situation that the related tasks have similar class labels. Zhang [2015] proposed the convex DMTRC algorithm which can model both positive and negative task correlations by learning the intertask covariance matrix of the multivariate Gaussian prior. Zhang and Zhang [2010, 2011] proposed a MBC, which alternatively updates the single-task Bregman clustering and learns the relationships between clusters of different tasks, and uses the two phases to boost each other. It aims to solve the multitask clustering problem when the related tasks are from a same distribution or similar distributions, such as slowly time-evolving data. And it has no need to assume that all tasks share an identical set of clusters. However, MBC may cause negative effects when the distributions of the tasks are not identical, and cannot solve multitask clustering problem of nonlinear separable data [Zhang and Zhang 2013]. In the preliminary version of this article [Zhang and Zhang 2013], we proposed a scheme to identify and avoid negative transfer of MBC, and an extension to deal with nonlinear separable data [Zhang and Zhang 2013].

\subsection{Transfer Learning}

Transfer learning [Pan and Yang 2010] is similar to multitask learning. It aims to utilize the knowledge learned from the source task to boost the performance of the target task. Whereas multitask learning focuses on boosting the performance of all the tasks. A lot of research work has been done in the field of inductive transfer leaning, transductive 
transfer learning and unsupervised transfer learning. In inductive transfer learning, there are some labeled data in the target domain, and there are a lot of labeled data [Dai et al. 2007b; Liao et al. 2005; Wu and Dietterich 2004] or no labeled data [Raina et al. 2007] in the source domain. In transductive transfer learning, there are no labeled data in the target domain, while a lot of labeled data in the source domain [Arnold et al. 2007; Dai et al. 2007a; Gu and Zhou 2009; Ling et al. 2008; Pan et al. 2008]. In unsupervised transfer learning, there are no labeled data in both the target and source domains [Dai et al. 2008; Jiang and Chung 2012].

\subsection{Negative Transfer}

Negative transfer has been specifically mentioned in Pan and Yang [2010], it is actually brought by the failure of solving "when to transfer" problem that asks in which situations the tasks can transfer knowledge. Negative transfer happens because when the tasks are dissimilar, brute-force transfer may decrease performance. There has been some work focusing on this topic. One way is to group tasks into several clusters in which the tasks in the same group are regarded as related [Bakker and Heskes 2003; Jacob et al. 2008; Romera-Paredes et al. 2012; Zhou et al. 2011]. The other way is to learn the intertask covariance matrix of the multivariate Gaussian prior which can model both positive and negative task correlations [Fei and Huan 2013; Saha et al. 2011; Zhang and Yeung 2010, 2012a, 2012b, 2014]. Zhang [2015] proposed the convex DMTRC algorithm based on the second way. Unlike the earlier methods, in the preliminary version of this article [Zhang and Zhang 2013], we proposed a step-by-step monitoring scheme to avoid negative transfer of the MBC algorithm.

\section{CONCLUSION}

In this article, we firstly observed that negative effects may occur in the previous $\mathrm{MBC}$ algorithm, and proposed the S-MBC algorithm which can identify and avoid such negative effects. Secondly, to deal with multitask clustering of nonlinear separable data, we proposed the MKC algorithm. We also propose a specific optimization method to implement the MKC framework. Furthermore, we showed that negative effects may also occur in MKC, and proposed the smart S-MKC to identify and avoid negative effects of MKC. Experimental results confirm our analysis and show that synthetically considering accuracy, efficiency, and applicability, our proposed algorithms S-MBC and S-MKC are competitive compared to the state-of-the-art algorithms. In future work, we will extend our MKC framework for general Bregman divergences and develop a unified method to identify and mitigate negative transfer.

\section{REFERENCES}

Rie K. Ando and Tong Zhang. 2005. A framework for learning predictive structures from multiple tasks and unlabeled data. Journal of Machine Learning Research 6, 1817-1853.

Andreas Argyriou, Theodoros Evgeniou, and Massimiliano Pontil. 2006. Multi-task feature learning. In Advances in Neural Information Processing Systems 19. Vancouver, British Columbia, Canada, 41-48.

Andrew Arnold, Ramesh Nallapati, and William W. Cohen. 2007. A comparative study of methods for transductive transfer learning. In Workshops Proceedings of the Seventh IEEE International Conference on Data Mining. Omaha, Nebraska, USA, 77-82.

Bart Bakker and Tom Heskes. 2003. Task clustering and gating for bayesian multitask learning. Journal of Machine Learning Research 4, 83-99.

Arindam Banerjee, Srujana Merugu, Inderjit S. Dhillon, and Joydeep Ghosh. 2005. Clustering with bregman divergences. Journal of Machine Learning Research 6, 1705-1749.

Edwin V. Bonilla, Kian Ming A. Chai, and Christopher K. I. Williams. 2007. Multi-task gaussian process prediction. In Advances in Neural Information Processing Systems 20. Vancouver, British Columbia, Canada, 153C160.

Stephen Boyd and Lieven Vandenberghe. 2004. Convex Optimization. Cambridge University Press, New York, NY, USA. 
Lev M. Bregman. 1967. The relaxation method of finding the common points of convex sets and its application to the solution of problems in convex programming. U. S. S. R. Comput. Math. and Math. Phys. 7, 3, 200-217.

Rich Caruana. 1997. Multitask learning. Machine Learning 28, 1, 41-75.

Jianhui Chen, Lei Tang, Jun Liu, and Jieping Ye. 2009. A convex formulation for learning shared structures from multiple tasks. In Proceedings of the Twenty-Sixth International Conference on Machine Learning. Montreal, Quebec, Canada, 137-144.

Wenyuan Dai, Gui-Rong Xue, Qiang Yang, and Yong Yu. 2007a. Co-clustering based classification for out-ofdomain documents. In Proceedings of the Thirteenth ACM SIGKDD International Conference on Knowledge Discovery and Data Mining. San Jose, California, USA, 210-219.

Wenyuan Dai, Qiang Yang, Gui-Rong Xue, and Yong Yu. 2007b. Boosting for transfer learning. In Proceedings of the Twenty-Fourth International Conference on Machine Learning. Corvalis, Oregon, USA, 193-200.

Wenyuan Dai, Qiang Yang, Gui-Rong Xue, and Yong Yu. 2008. Self-taught clustering. In Proceedings of the Twenty-Fifth International Conference on Machine Learning. Helsinki, Finland, 200-207.

Inderjit S. Dhillon and Suvrit Sra. 2005. Generalized nonnegative matrix approximations with bregman divergences. In Advances in Neural Information Processing Systems 18. Vancouver, British Columbia, Canada

Chris H. Q. Ding, Tao Li, and Michael I. Jordan. 2010. Convex and semi-nonnegative matrix factorizations. IEEE Transactions on Pattern Analysis and Machine Intelligence 32, 1, 45-55.

Theodoros Evgeniou, Charles A. Micchelli, and Massimiliano Pontil. 2005. Learning multiple tasks with kernel methods. Journal of Machine Learning Research 6, 615-637.

Theodoros Evgeniou and Massimiliano Pontil. 2004. Regularized multi-task learning. In Proceedings of the Tenth ACM SIGKDD International Conference on Knowledge Discovery and Data Mining. Seattle, Washington, USA, 109-117.

Hongliang Fei and Jun Huan. 2013. Structured feature selection and task relationship inference for multitask learning. Knowledge and Information Systems 35, 2, 345-364.

Quanquan $\mathrm{Gu}$, Zhenhui Li, and Jiawei Han. 2011. Learning a kernel for multi-task clustering. In Proceedings of the Twenty-Fifth AAAI Conference on Artificial Intelligence. San Francisco, California, USA, 368-373.

Quanquan Gu and Jie Zhou. 2009. Learning the shared subspace for multi-task clustering and transductive transfer classification. In Proceedings of the Ninth IEEE International Conference on Data Mining. Miami, Florida, USA, 159-168.

Laurent Jacob, Francis Bach, and Jean-Philippe Vert. 2008. Clustered multi-task learning: A convex formulation. In Advances in Neural Information Processing Systems 21. Vancouver, British Columbia, Canada, $745-752$.

Wenhao Jiang and Fu-Lai Chung. 2012. Transfer spectral clustering. In Proceedings of European Conference on Machine Learning and Knowledge Discovery in Databases Part II. Bristol, UK, 789-803.

Neil D. Lawrence and John C. Platt. 2004. Learning to learn with the informative vector machine. In Proceedings of the Twenty-First International Conference on Machine Learning. Banff, Alberta, Canada.

Xuejun Liao, Ya Xue, and Lawrence Carin. 2005. Logistic regression with an auxiliary data source. In Proceedings of the Twenty-Second International Conference on Machine Learning. Bonn, Germany, 505512.

Xiao Ling, Wenyuan Dai, Gui-Rong Xue, Qiang Yang, and Yong Yu. 2008. Spectral domain-transfer learning. In Proceedings of the Fourteenth ACM SIGKDD International Conference on Knowledge Discovery and Data Mining. Las Vegas, Nevada, USA, 488-496.

Charles A. Micchelli and Massimiliano Pontil. 2004. Kernels for Multi-task Learning. In Advances in Neural Information Processing Systems 17. Vancouver, British Columbia, Canada.

Morten Mørup and Lars Kai Hansen. 2009. An Exact Relaxation of Clustering. Technical Report. Technical University of Denmark.

Thach Huy Nguyen, Hao Shao, Bin Tong, and Einoshin Suzuki. 2011. A compression-based dissimilarity measure for multi-task clustering. In Proceedings of the Nineteenth International Symposium on Methodologies for Intelligent Systems. Warsaw, Poland, 123-132.

Thach Huy Nguyen, Hao Shao, Bin Tong, and Einoshin Suzuki. 2013. A feature-free and parameter-light multi-task clustering framework. Knowledge and Information Systems 36, 1, 251-276.

Frank Nielsen and Richard Nock. 2009. Sided and symmetrized bregman centroids. IEEE Transactions on Information Theory 55, 6, 2882-2904.

Sinno Jialin Pan, James T. Kwok, and Qiang Yang. 2008. Transfer learning via dimensionality reduction. In Proceedings of the Twenty-Third AAAI Conference on Artificial Intelligence. Chicago, Illinois, USA, $677-682$. 
Sinno Jialin Pan and Qiang Yang. 2010. A survey on transfer learning. IEEE Transactions on Knowledge and Data Engineering 22, 10, 1345-1359.

Rajat Raina, Alexis Battle, Honglak Lee, Benjamin Packer, and Andrew Y. Ng. 2007. Self-taught learning: Transfer learning from unlabeled data. In Proceedings of the Twenty-Fourth International Conference on Machine Learning. Corvalis, Oregon, USA, 759-766.

Bernardino Romera-Paredes, Andreas Argyriou, Nadia Berthouze, and Massimiliano Pontil. 2012. Exploiting unrelated tasks in multi-task learning. In Proceedings of the Fifteenth International Conference on Artificial Intelligence and Statistics. La Palma, Canary Islands, 951-959.

Yossi Rubner, Carlo Tomasi, and Leonidas J. Guibas. 1998. A metric for distributions with applications to image databases. In Proceedings of the Sixth International Conference on Computer Vision. Bombay, India, 59-66.

Avishek Saha, Piyush Rai, Hal Daumé III, and Suresh Venkatasubramanian. 2011. Online learning of multiple tasks and their relationships. In Proceedings of the Fourteenth International Conference on Artificial Intelligence and Statistics. Fort Lauderdale, USA, 643-651.

Craig Saunders, Mark O. Stitson, Jason Weston, Léon Bottou, Bernhard Schölkopf, and Alexander J. Smola. 1998. Support Vector Machine Reference Manual. Technical Report CSD-TR-98-03. Royal Holloway College, University of London.

John Shawe-Taylor and Nello Cristianini. 2004. Kernel Methods for Pattern Analysis. Cambridge University Press. I-XIV, 1-462 pages.

Pengcheng Wu and Thomas G. Dietterich. 2004. Improving SVM accuracy by training on auxiliary data sources. In Proceedings of the Twenty-First International Conference on Machine Learning. Banff, Alberta, Canada.

Saining Xie, Hongtao Lu, and Yangcheng He. 2012. Multi-task co-clustering via nonnegative matrix factorization. In Proceedings of the Twenty-First International Conference on Pattern Recognition. Tsukuba, Japan, 2954-2958.

Wei Xu, Xin Liu, and Yihong Gong. 2003. Document clustering based on non-negative matrix factorization. In Proceedings of the Twenty-Sixth International ACM SIGIR Conference on Research and Development in Information Retrieval. Toronto, Canada, 267-273.

Jianwen Zhang and Changshui Zhang. 2010. Multitask bregman clustering. In Proceedings of the TwentyFourth AAAI Conference on Artificial Intelligence. Atlanta, Georgia, USA.

Jianwen Zhang and Changshui Zhang. 2011. Multitask bregman clustering. Neurocomputing 74, 10, 17201734.

Xiao-Lei Zhang. 2015. Convex discriminative multitask clustering. IEEE Transactions on Pattern Analysis and Machine Intelligence 37, 1, 28-40.

Xianchao Zhang and Xiaotong Zhang. 2013. Smart multi-task bregman clustering and multi-task kernel clustering. In Proceedings of the Twenty-Seventh AAAI Conference on Artificial Intelligence. Bellevue, Washington, USA, 1034-1040.

Yin Zhang. 1996. Solving Large-Scale Linear Programs by Interior-Point Methods Under the MATLAB Environment. Technical Report TR96-01. Department of Mathematics and Statistics, University of Maryland Baltimore County.

Yu Zhang and Dit-Yan Yeung. 2010. A convex formulation for learning task relationships in multi-task learning. In Proceedings of the Twenty-Sixth Conference on Uncertainty in Artificial Intelligence. Catalina Island, CA, USA, 733-442.

Yu Zhang and Dit-Yan Yeung. 2012a. Multi-task boosting by exploiting task relationships. In Proceedings of European Conference on Machine Learning and Knowledge Discovery in Databases Part I. Bristol, UK, 697-710.

Yu Zhang and Dit-Yan Yeung. 2012b. Transfer metric learning with semi-supervised extension. ACM Transactions on Intelligent Systems and Technology 3, 3.

Yu Zhang and Dit-Yan Yeung. 2014. A regularization approach to learning task relationships in multi-task learning. ACM Transactions on Knowledge Discovery from Data, accepted.

Zhihao Zhang and Jie Zhou. 2012. Multi-task clustering via domain adaptation. Pattern Recognition 45, 1, $465-473$.

Shi Zhong and Joydeep Ghosh. 2003. A unified framework for model-based clustering. Journal of Machine Learning Research 4, 1001-1037.

Jiayu Zhou, Jianhui Chen, and Jieping Ye. 2011. Clustered multi-task learning via alternating structure optimization. In Advances in Neural Information Processing Systems 24. Granada, Spain, 702-710.

Received July 2014; revised December 2014; accepted March 2015 